\title{
ADDING A LOT OF COHEN REALS BY ADDING A FEW. I
}

\author{
MOTI GITIK AND MOHAMMAD GOLSHANI
}

ABSTRACT. In this paper we produce models $V_{1} \subseteq V_{2}$ of set theory such that adding $\kappa$-many Cohen reals to $V_{2}$ adds $\lambda$-many Cohen reals to $V_{1}$, for some $\lambda>\kappa$. We deal mainly with the case when $V_{1}$ and $V_{2}$ have the same cardinals.

\section{INTRODUCTION}

A basic fact about Cohen reals is that adding $\lambda$-many Cohen reals cannot produce more than $\lambda$-many Cohen reals 1 More precisely, if $\left\langle s_{\alpha}: \alpha<\lambda\right\rangle$ are $\lambda$-many Cohen reals over $V$, then in $V\left[\left\langle s_{\alpha}: \alpha<\lambda\right\rangle\right]$ there are no $\lambda^{+}$-many Cohen reals over $V$. But if instead of dealing with one universe $V$ we consider two; then the above may no longer be true.

The purpose of this paper is to produce models $V_{1} \subseteq V_{2}$ such that adding $\kappa$-many Cohen reals to $V_{2}$ adds $\lambda$-many Cohen reals to $V_{1}$, for some $\lambda>\kappa$. We deal mainly with the case when $V_{1}$ and $V_{2}$ have the same cardinals.

\section{Models With The SAME REALS}

In this section we produce models $V_{1} \subseteq V_{2}$ as above with the same reals. We first state a general result.

Theorem 2.1. Let $V_{1}$ be an extension of $V$. Suppose that in $V_{1}$ :

(a) $\kappa<\lambda$ are infinite cardinals,

(b) $\lambda$ is regular,

(c) there exists an increasing sequence $\left\langle\kappa_{n}: n<\omega\right\rangle$ of regular cardinals cofinal in $\kappa$; in particular, $\operatorname{cf}(\kappa)=\omega$,

$(d)$ there exists an increasing (mod finite) sequence $\left\langle f_{\alpha}: \alpha<\lambda\right\rangle$ of functions in $\prod_{n<\omega}\left(\kappa_{n+1} \backslash \kappa_{n}\right) 2$ and

(e) there exists a club $C \subseteq \lambda$ which avoids points of countable $V$-cofinality.

Then adding $\kappa$-many Cohen reals over $V_{1}$ produces $\lambda$-many Cohen reals over $V$.

Proof. We consider two cases.

Case $\lambda=\kappa^{+}$. Force to add $\kappa$-many Cohen reals over $V_{1}$. Split them into two sequences of length $\kappa$ denoted by $\left\langle r_{\imath}: \imath<\kappa\right\rangle$ and $\left\langle r_{\imath}^{\prime}: \imath<\kappa\right\rangle$. Also let

Received by the editors August 19, 2011 and, in revised form, November 9, 2012.

2010 Mathematics Subject Classification. Primary 03E35.

The second author would like to thank the Austrian Science Fund (FWF) for its support through research project P 223316-N13.

${ }^{1}$ By " $\lambda$-many Cohen reals" we mean "a generic object $\left\langle s_{\alpha}: \alpha<\lambda\right\rangle$ for the poset $\mathbb{C}(\lambda)$ of finite partial functions from $\lambda \times \omega$ to 2 ".

${ }^{2}$ Note that condition $(d)$ holds automotically for $\lambda=\kappa^{+}$; given any collection $\mathcal{F}$ of $\kappa$-many elements of $\prod_{n<\omega}\left(\kappa_{n+1} \backslash \kappa_{n}\right)$ there exists $f$ such that for each $g \in \mathcal{F}, f(n)>g(n)$ for all large $n$. Thus we can define, by induction on $\alpha<\kappa^{+}$, an increasing (mod finite) sequence $\left\langle f_{\alpha}: \alpha<\kappa^{+}\right\rangle$ in $\prod_{n<\omega}\left(\kappa_{n+1} \backslash \kappa_{n}\right)$. 
$\left\langle f_{\alpha}: \alpha<\kappa^{+}\right\rangle \in V_{1}$ be an increasing (mod finite) sequence in $\prod_{n<\omega}\left(\kappa_{n+1} \backslash \kappa_{n}\right)$. Let $\alpha<\kappa^{+}$. We define a real $s_{\alpha}$ as follows:

Case 1. $\alpha \in C$. Then

$$
\forall n<\omega, s_{\alpha}(n)=r_{f_{\alpha}(n)}(0) .
$$

Case 2. $\alpha \notin C$. Let $\alpha^{*}$ and $\alpha^{* *}$ be two successive points of $C$ so that $\alpha^{*}<\alpha<\alpha^{* *}$. Let $\left\langle\alpha_{\imath}: \imath<\kappa\right\rangle$ be some fixed enumeration of the interval $\left(\alpha^{*}, \alpha^{* *}\right)$. Then for some $\imath<\kappa, \alpha=\alpha_{\imath}$. Let $k(\imath)=\min \left\{k<\omega: r_{\imath}^{\prime}(k)=1\right\}$. Set

$$
\forall n<\omega, s_{\alpha}(n)=r_{f_{\alpha}(k(\imath)+n)}(0) .
$$

The following lemma completes the proof.

Lemma 2.2. $\left\langle s_{\alpha}: \alpha<\kappa^{+}\right\rangle$is a sequence of $\kappa^{+}$-many Cohen reals over $V$.

Notation 2.3. For each set $I$, let $\mathbb{C}(I)$ be the Cohen forcing notion for adding $I$ many Cohen reals. Thus $\mathbb{C}(I)=\{p: p$ is a finite partial function from $I \times \omega$ into 2$\}$, ordered by reverse inclusion.

Proof. First note that $\left\langle\left\langle r_{\imath}: \imath<\kappa\right\rangle,\left\langle r_{\imath}^{\prime}: \imath<\kappa\right\rangle\right\rangle$ is $\mathbb{C}(\kappa) \times \mathbb{C}(\kappa)$-generic over $V_{1}$. By the c.c.c. of $\mathbb{C}\left(\kappa^{+}\right)$it suffices to show that for any countable set $I \subseteq \kappa^{+}, I \in V$, the sequence $\left\langle s_{\alpha}: \alpha \in I\right\rangle$ is $\mathbb{C}(I)$-generic over $V$. Thus it suffices to prove the following:

$$
\text { for every }(p, q) \in \mathbb{C}(\kappa) \times \mathbb{C}(\kappa) \text { and every open dense }
$$

$$
\begin{aligned}
& \text { subset } D \in V \text { of } \mathbb{C}(I) \text {, there is }(\bar{p}, \bar{q}) \leq(p, q) \text { such that } \\
& (\bar{p}, \bar{q}) \|-"\left\langle\sim_{\sim}^{s} \alpha: \alpha \in I\right\rangle \text { extends some element of } D " .
\end{aligned}
$$

Let $(p, q)$ and $D$ be as above. For simplicity suppose that $p=q=\emptyset$. By $(e)$ there are only finitely many $\alpha^{*} \in C$ such that $I \cap\left[\alpha^{*}, \alpha^{* *}\right) \neq \emptyset$, where $\alpha^{* *}=$ $\min \left(C \backslash\left(\alpha^{*}+1\right)\right)$. For simplicity suppose that there are two $\alpha_{1}^{*}<\alpha_{2}^{*}$ in $C$ with this property. Let $n^{*}<\omega$ be such that for all $n \geq n^{*}, f_{\alpha_{1}^{*}}(n)<f_{\alpha_{2}^{*}}(n)$. Let $p \in \mathbb{C}(\kappa)$ be such that

$$
\operatorname{dom}(p)=\left\{\langle\beta, 0\rangle: \exists n<n^{*}\left(\beta=f_{\alpha_{1}^{*}}(n) \text { or } \beta=f_{\alpha_{2}^{*}}(n)\right)\right\} .
$$

Then for $n<n^{*}$ and $j \in\{1,2\}$,

$$
(p, \emptyset) \|-\underset{\sim}{\sim} \alpha_{j}^{*}(n)=\underset{\sim}{\sim} f_{\alpha_{j}^{*}}(n)=p\left(f_{\alpha_{j}^{*}(n)}, 0\right) .
$$

Thus $(p, \emptyset)$ decides $s_{\alpha_{1}^{*}}\left\lceil n^{*}\right.$ and $s_{\alpha_{2}^{*}}\left\lceil n^{*}\right.$. Let $b \in D$ be such that $\left\langle b\left(\alpha_{1}^{*}\right), b\left(\alpha_{2}^{*}\right)\right\rangle$ ex-

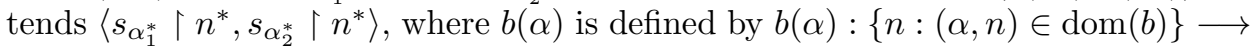
2 and $b(\alpha)(n)=b(\alpha, n)$. Let

$$
p^{\prime}=p \cup \bigcup_{j \in\{1,2\}}\left\{\left\langle f_{\alpha_{j}^{*}}(n), 0, b\left(\alpha_{j}^{*}, n\right)\right\rangle: n \geq n^{*},\left(\alpha_{j}^{*}, n\right) \in \operatorname{dom}(b)\right\} .
$$

Then $p^{\prime} \in \mathbb{C}(\kappa)^{3}$ and

$$
\left(p^{\prime}, \emptyset\right) \|-"\left\langle\underset{\sim}{s} \alpha_{1}^{*}, \underset{\sim}{s} \alpha_{2}^{*}\right\rangle \quad \text { extends }\left\langle b\left(\alpha_{1}^{*}\right), b\left(\alpha_{2}^{*}\right)\right\rangle " .
$$

\footnotetext{
${ }^{3}$ This is because for $n \geq n^{*}, f_{\alpha_{1}^{*}}(n) \neq f_{\alpha_{2}^{*}}(n)$, and for $j \in\{1,2\}, f_{\alpha_{j}^{*}}(n) \notin\left\{f_{\alpha_{j}^{*}}(m): m<n\right\}$; thus there are no collisions.
} 
For $j \in\{1,2\}$, let $\left\{\alpha_{j_{0}}, \ldots, \alpha_{j k_{j}-1}\right\}$ be an increasing enumeration of components of $b$ in the interval $\left(\alpha_{j}^{*}, \alpha_{j}^{* *}\right)$ (i.e. those $\alpha \in\left(\alpha_{j}^{*}, \alpha_{j}^{* *}\right)$ such that $(\alpha, n) \in \operatorname{dom}(b)$ for some $n)$. For $j \in\{1,2\}$ and $l<k_{j}$ let $\alpha_{j l}=\alpha_{\imath_{j l}}$, where $\imath_{j l}<\kappa$ is the index of $\alpha_{j l}$ in the enumeration of the interval $\left(\alpha_{j}^{*}, \alpha_{j}^{* *}\right)$ considered in Case 2 above. Let $m^{*}<\omega$ be such that for all $n \geq m^{*}, j \in\{1,2\}$ and $l_{j}<l_{j}^{\prime}<k_{j}$ we have

$$
f_{\alpha_{1}^{*}}(n)<f_{\alpha_{1 \ell_{1}}}(n)<f_{\alpha_{1 \ell_{1}^{\prime}}}(n)<f_{\alpha_{2}^{*}}(n)<f_{\alpha_{2 \ell_{2}}}(n)<f_{\alpha_{2 \ell_{2}^{\prime}}}(n) .
$$

Let

$$
\bar{q}=\left\{\left\langle\imath_{j l}, n, 0\right\rangle: j \in\{1,2\}, l<k_{j}, n<m^{*}\right\} \cup\left\{\left\langle\imath_{j l}, m^{*}, 1\right\rangle: j \in\{1,2\}, l<k_{j}\right\} .
$$

Then $\bar{q} \in \mathbb{C}(\kappa)$ and for $j \in\{1,2\}$ and $n<m^{*},(\emptyset, \bar{q}) \|-{ }^{\prime \prime} r_{\imath_{j l}}^{\prime}(n)=0$ and $r_{\imath_{j l}}^{\prime}\left(m^{*}\right)=$ $1 "$; thus $(\emptyset, \bar{q}) \|-k(j, l)=\min \left\{k<\omega: r_{\imath_{j l}}^{\prime}(k)=1\right\}=m^{*}$. Let

$$
\bar{p}=p^{\prime} \cup \bigcup_{j \in\{1,2\}}\left\{\left\langle f_{\alpha_{j l}}\left(m^{*}+n\right), 0, b\left(\alpha_{j l}, n\right)\right\rangle: l<k_{j},\left(\alpha_{j l}, n\right) \in \operatorname{dom}(b)\right\} .
$$

It is easily seen that $\bar{p} \in \mathbb{C}(\kappa)$ is well defined, and for $j \in\{1,2\}$ and $l<k_{j}$,

$$
(\bar{p}, \bar{q}) \|-" \stackrel{s}{\sim} \alpha_{j l} \text { extends } b\left(\alpha_{j l}\right) " .
$$

Thus

$$
(\bar{p}, \bar{q}) \|-"\langle\underset{\sim}{s} \alpha: \alpha \in I\rangle \text { extends } b " .
$$

$(*)$ follows and we are done.

Case $\lambda>\kappa^{+}$. Force to add $\kappa$-many Cohen reals over $V_{1}$. We now construct $\lambda$-many Cohen reals over $V$ as in the above case using $C$ and $\left\langle f_{\alpha}: \alpha<\lambda\right\rangle$. Case 2 of the definition of $\left\langle s_{\alpha}: \alpha<\lambda\right\rangle$ is now problematic since the cardinality of an interval $\left(\alpha^{*}, \alpha^{* *}\right)$ (using the above notation) may now be above $\kappa$ and we have only $\kappa$-many Cohen reals to play with. Let us proceed as follows in order to overcome this.

Let us rearrange the Cohen reals as $\left\langle r_{n, \alpha}: n<\omega, \alpha<\kappa\right\rangle$ and $\left\langle r_{\eta}: \eta \in[\kappa]^{<\omega}\right\rangle$. We define by induction on levels a tree $T \subseteq[\lambda]^{<\omega}$, its projection $\pi(T) \subseteq[\kappa]^{<\omega}$, and for each $n<\omega$ and $\alpha \in \operatorname{Lev}_{n}(T)$, a real $s_{\alpha}$. The union of the levels of $T$ will be $\lambda$, so $\left\langle s_{\alpha}: \alpha<\lambda\right\rangle$ will be defined.

For $n=0$, let $\operatorname{Lev}_{0}(T)=\langle\rangle=\operatorname{Lev}_{0}(\pi(T))$.

For $n=1$, let $\operatorname{Lev}_{1}(T)=C, \operatorname{Lev}_{1}(\pi(T))=\{0\}$, i.e. $\pi(\langle\alpha\rangle)=\langle 0\rangle$ for every $\alpha \in C$. For $\alpha \in C$ we define a real $s_{\alpha}$ by

$$
\forall m<\omega, s_{\alpha}(m)=r_{1, f_{\alpha}(m)}(0) .
$$

Suppose now that $n>1$ and $T\left\lceil n\right.$ and $\pi(T) \uparrow n$ are defined. We define $\operatorname{Lev}_{n}(T)$, $\operatorname{Lev}_{n}(\pi(T))$ and reals $s_{\alpha}$ for $\alpha \in \operatorname{Lev}_{n}(T)$. Let $\eta \in T \uparrow(n-1), \alpha^{*}, \alpha^{* *} \in \operatorname{Suc}_{T}(\eta)$ and $\alpha^{* *}=\min \left(S u c_{T}(\eta) \backslash\left(\alpha^{*}+1\right)\right)$. We then define $S u c_{T}\left(\eta \frown\left\langle\alpha^{* *}\right\rangle\right)$ if it is not yet defined $\sqrt{4}$

Case A. $\left|\alpha^{* *} \backslash \alpha^{*}\right| \leq \kappa$.

Fix some enumeration $\left\langle\alpha_{\imath}: \imath<\rho \leq \kappa\right\rangle$ of $\alpha^{* *} \backslash \alpha^{*}$. Let

- $\operatorname{Suc}_{T}\left(\eta \frown\left\langle\alpha^{* *}\right\rangle\right)=\alpha^{* *} \backslash \alpha^{*}$

- $\operatorname{Suc}_{T}\left(\eta \frown\left\langle\alpha^{* *}\right\rangle \frown\langle\alpha\rangle\right)=\langle\rangle$ for $\alpha \in \alpha^{* *} \backslash \alpha^{*}$,

\footnotetext{
${ }^{4}$ Then $\operatorname{Lev}_{n}(T)$ will be the union of such $S u c_{T}\left(\eta \frown\left\langle\alpha^{* *}\right\rangle\right)$ 's.
} 
- $\operatorname{Suc}_{\pi(T)}\left(\pi\left(\eta \frown\left\langle\alpha^{* *}\right\rangle\right)\right)=\rho=\left|\alpha^{* *} \backslash \alpha^{*}\right|$,

- $\operatorname{Suc}_{\pi(T)}\left(\pi\left(\eta \frown\left\langle\alpha^{* *}\right\rangle\right) \frown\langle\imath\rangle\right)=\langle\rangle$ for $\imath<\rho$.

Now we define $s_{\alpha}$ for $\alpha \in \alpha^{* *} \backslash \alpha^{*}$. Let $\imath$ be such that $\alpha=\alpha_{\imath}$. Let $k=$ $\min \left\{m<\omega: r_{\pi\left(\eta-\left\langle\alpha^{* *}\right\rangle\right) \frown\langle\imath\rangle}(m)=1\right\}$, Finally let

$$
\forall m<\omega, s_{\alpha}(m)=r_{n, f_{\alpha}(k+m)}(0) .
$$

Case B. $\left|\alpha^{* *} \backslash \alpha^{*}\right|>\kappa$ and $\operatorname{cf}\left(\alpha^{* *}\right)<\kappa$.

Let $\rho=c f \alpha^{* *}$ and let $\left\langle\alpha_{\nu}^{* *}: \nu<\rho\right\rangle$ be a normal sequence cofinal in $\alpha^{* *}$ with $\alpha_{0}^{* *}>\alpha^{*}$. Let

- $\operatorname{Suc}_{T}\left(\eta \frown\left\langle\alpha^{* *}\right\rangle\right)=\left\{\alpha_{\nu}^{* *}: \nu<\rho\right\}$,

- $\operatorname{Suc}_{\pi(T)}\left(\pi\left(\eta \frown\left\langle\alpha^{* *}\right\rangle\right)\right)=\rho$.

Now we define $s_{\alpha_{\nu}^{* *}}$ for $\nu<\rho$. Let $k=\min \left\{m<\omega: r_{\pi\left(\eta-\left\langle\alpha^{* *}\right\rangle\right)-\langle\nu\rangle}(m)=1\right\}$ and let

$$
\forall m<\omega, s_{\alpha_{\nu}^{* *}}(m)=r_{n, f_{\alpha_{\nu}^{* *}}(k+m)}(0) .
$$

Case C. $c f\left(\alpha^{* *}\right)>\kappa$.

Let $\rho$ and $\left\langle\alpha_{\nu}^{* *}: \nu<\rho\right\rangle$ be as in Case B. Let

- $\operatorname{Suc}_{T}\left(\eta \frown\left\langle\alpha^{* *}\right\rangle\right)=\left\{\alpha_{\nu}^{* *}: \nu<\rho\right\}$,

- $\operatorname{Suc}_{\pi(T)}\left(\pi\left(\eta \frown\left\langle\alpha^{* *}\right\rangle\right)\right)=\langle 0\rangle$.

We define $s_{\alpha_{\nu}^{* *}}$ for $\nu<\rho$. Let $k=\min \left\{m<\omega: r_{\pi\left(\eta-\left\langle\alpha^{* *}\right\rangle\right) \frown\langle 0\rangle}(m)=1\right\}$ and let

$$
\forall m<\omega, s_{\alpha_{\nu}^{* *}}(m)=r_{n, f_{\alpha_{\nu}^{* *}}(k+m)}(0) .
$$

By the definition, $T$ is a well-founded tree and $\bigcup_{n<\omega} \operatorname{Lev} v_{n}(T)=\lambda$. The following lemma completes our proof.

Lemma 2.4. $\left\langle s_{\alpha}: \alpha<\lambda\right\rangle$ is a sequence of $\lambda$-many Cohen reals over $V$.

Proof. First note that $\left\langle\left\langle r_{n, \alpha}: n<\omega, \alpha<\kappa\right\rangle,\left\langle r_{\eta}: \eta \in[\kappa]<\omega\right\rangle\right\rangle$ is $\mathbb{C}(\omega \times \kappa) \times$ $\mathbb{C}\left([\kappa]^{<\omega}\right)$-generic over $V_{1}$. By the c.c.c. of $\mathbb{C}(\lambda)$ it suffices to show that for any countable set $I \subseteq \lambda, I \in V$, the sequence $\left\langle s_{\alpha}: \alpha \in I\right\rangle$ is $\mathbb{C}(I)$-generic over $V$. Thus it suffices to prove the following:

For every $(p, q) \in \mathbb{C}(\omega \times \kappa) \times \mathbb{C}\left([\kappa]^{<\omega}\right)$ and every open dense subset $D \in V$ of $\mathbb{C}(I)$, there is $(\bar{p}, \bar{q}) \leq(p, q)$ such that $(\bar{p}, \bar{q}) \|-$ " $\underset{\sim}{\sim} \alpha: \alpha \in I\rangle$ extends some element of $D$ ".

Let $(p, q)$ and $D$ be as above. For simplicity suppose that $p=q=\emptyset$. For each $n<\omega$ let $I_{n}=I \cap \operatorname{Lev}_{n}(T)$. Then $I_{0}=\emptyset$ and $I_{1}=I \cap C$ is finite. For simplicity let $I_{1}=\left\{\alpha_{1}^{*}, \alpha_{2}^{*}\right\}$, where $\alpha_{1}^{*}<\alpha_{2}^{*}$. Pick $n^{*}<\omega$ such that for all $n \geq n^{*}$, $f_{\alpha_{1}^{*}}(n)<f_{\alpha_{2}^{*}}(n)$. Let $p_{0} \in \mathbb{C}(\omega \times \kappa)$ be such that

$$
\operatorname{dom}\left(p_{0}\right)=\left\{\langle 1, \beta, 0\rangle: \exists n<n^{*}\left(\beta=f_{\alpha_{1}^{*}}(n) \text { or } \beta=f_{\alpha_{2}^{*}}(n)\right)\right\} .
$$

Then for $n<n^{*}$ and $j \in\{1,2\}$,

$$
\left(p_{0}, \emptyset\right) \|-\underset{\sim}{\sim} \alpha_{j}^{*}(n)={\underset{\sim}{\sim} 1, f_{\alpha_{j}^{*}}(n)}(0)=p_{0}\left(1, f_{\alpha_{j}^{*}}(n), 0\right) .
$$


Thus $\left(p_{0}, \emptyset\right)$ decides $s_{\alpha_{1}^{*}}\left\lceil n^{*}\right.$ and $s_{\alpha_{2}^{*}}\left\lceil n^{*}\right.$. Let $b \in D$ be such that $\left\langle b\left(\alpha_{1}^{*}\right), b\left(\alpha_{2}^{*}\right)\right\rangle$ extends $\left\langle s_{\alpha_{1}^{*}}\left\lceil n^{*}, s_{\alpha_{2}^{*}}\left\lceil n^{*}\right\rangle\right.\right.$. Let

$$
p_{1}=p_{0} \cup \bigcup_{j \in\{1,2\}}\left\{\left\langle 1, f_{\alpha_{j}^{*}}(n), 0, b\left(\alpha_{j}^{*}, n\right)\right\rangle: n \geq n^{*},\left(\alpha_{j}^{*}, n\right) \in \operatorname{dom}(b)\right\} .
$$

Then $p_{1} \in \mathbb{C}(\omega \times \kappa)$ is well defined, and letting $q_{1}=\emptyset$, we have

$$
\left(p_{1}, q_{1}\right) \|-"\left\langle\underset{\sim}{s} \alpha_{1}^{*}, \underset{\sim}{s} \alpha_{2}^{*}\right\rangle \text { extends }\left\langle b\left(\alpha_{1}^{*}\right), b\left(\alpha_{2}^{*}\right)\right\rangle " .
$$

For each $n<\omega$ let $J_{n}$ be the set of all components of $b$ which are in $I_{n}$, i.e. $J_{n}=\left\{\alpha \in I_{n}: \exists n,(\alpha, n) \in \operatorname{dom}(b)\right\}$. We note that $J_{0}=\emptyset$ and $J_{1}=I_{1}=\left\{\alpha_{1}^{*}, \alpha_{2}^{*}\right\}$. Also note that for all but finitely many $n<\omega, J_{n}=\emptyset$. Thus let us suppose $t<\omega$ is such that for all $n>t, J_{n}=\emptyset$. Let us consider $J_{2}$. For each $\alpha \in J_{2}$ there are three cases to be considered 5

Case 1. There are $\alpha^{*}<\alpha^{* *}$ in $\operatorname{Lev}_{1}(T)=C, \alpha^{* *}=\min \left(C \backslash\left(\alpha^{*}+1\right)\right)$ such that $\left|\alpha^{* *} \backslash \alpha^{*}\right| \leq \kappa$ and $\alpha \in \operatorname{Suc}_{T}\left(\left\langle\alpha^{* *}\right\rangle\right)=\alpha^{* *} \backslash \alpha^{*}$. Let $\imath_{\alpha}$ be the index of $\alpha$ in the enumeration of $\alpha^{* *} \backslash \alpha^{*}$ considered in Case A above, and let $k_{\alpha}=\min \{m<\omega$ : $\left.r_{\pi\left(\left\langle\alpha^{* *}\right\rangle\right)-\left\langle\imath_{\alpha}\right\rangle}(m)=1\right\}$. Then

$$
\forall m<\omega, s_{\alpha}(m)=r_{2, f_{\alpha}\left(k_{\alpha}+m\right)}(0) .
$$

Case 2. There are $\alpha^{*}<\alpha^{* *}$ as above such that $\left|\alpha^{* *} \backslash \alpha^{*}\right|>\kappa$ and $\rho=c f \alpha^{* *}<\kappa$. Let $\left\langle\alpha_{\nu}^{* *}: \nu<\rho\right\rangle$ be as in Case B. Then $\alpha=\alpha_{\nu_{\alpha}}^{* *}$ for some $\nu_{\alpha}<\rho$ and if $k_{\alpha}=$ $\min \left\{m<\omega: r_{\pi\left(\left\langle\alpha^{* *}\right\rangle\right)-\left\langle\nu_{\alpha}\right\rangle}(m)=1\right\}$. Then

$$
\forall m<\omega, s_{\alpha}(m)=r_{2, f_{\alpha}\left(k_{\alpha}+m\right)}(0) .
$$

Case 3. There are $\alpha^{*}<\alpha^{* *}$ as above such that $\rho=\operatorname{cf} \alpha^{* *}>\kappa$. Let $\left\langle\alpha_{\nu}^{* *}: \nu<\rho\right\rangle$ be as in Case C. Then $\alpha=\alpha_{\nu_{\alpha}}^{* *}$ for some $\nu_{\alpha}<\rho$, and if $k_{\alpha}=\min \{m<\omega$ : $\left.r_{\pi\left(\left\langle\alpha^{* *}\right\rangle\right) \frown\langle 0\rangle}(m)=1\right\}$, then

$$
\forall m<\omega, s_{\alpha}(m)=r_{2, f_{\alpha}\left(k_{\alpha}+m\right)}(0) .
$$
Let

Let $m^{*}<\omega$ be such that for all $n \geq m^{*}$ and $\alpha<\alpha^{\prime}$ in $J_{1} \cup J_{2}, f_{\alpha}(n)<f_{\alpha^{\prime}}(n)$.

$$
\begin{aligned}
& q_{2}=\left\{\langle\eta, n, 0\rangle: n<m^{*}, \exists \alpha \in J_{2}\left(\eta=\pi\left(\left\langle\alpha^{* *}\right\rangle\right) \frown\left\langle i_{\alpha}\right\rangle\right. \text { or }\right. \\
& \eta=\pi\left(\left\langle\alpha^{* *}\right\rangle\right) \frown\left\langle\nu_{\alpha}\right\rangle \text { or } \\
& \left.\left.\eta=\pi\left(\left\langle\alpha^{* *}\right\rangle\right) \frown\langle 0\rangle\right)\right\} \\
& \cup\left\{\left\langle\eta, m^{*}, 1\right\rangle: \exists \alpha \in J_{2}\left(\eta=\pi\left(\left\langle\alpha^{* *}\right\rangle\right) \frown\left\langle i_{\alpha}\right\rangle\right. \text { or }\right. \\
& \eta=\pi\left(\left\langle\alpha^{* *}\right\rangle\right) \frown\left\langle\nu_{\alpha}\right\rangle \text { or } \\
& \left.\left.\eta=\pi\left(\left\langle\alpha^{* *}\right\rangle\right) \frown\langle 0\rangle\right)\right\} .
\end{aligned}
$$

Then $q_{2} \in \mathbb{C}\left([\kappa]^{<\omega}\right)$ is well defined, and for each $\alpha \in J_{2},\left(\phi, q_{2}\right) \|-k_{\alpha}=m^{*}$. Let

$$
p_{2}=p_{1} \cup\left\{\left\langle 2, f_{\alpha}\left(m^{*}+m\right), 0, b(\alpha, m)\right\rangle: \alpha \in J_{2},(\alpha, m) \in \operatorname{dom}(b)\right\} .
$$

Then $p_{2} \in \mathbb{C}(\omega \times \kappa)$ is well defined, $\left(p_{2}, q_{2}\right) \leq\left(p_{1}, q_{1}\right)$, and for $\alpha \in J_{2}$ and $m<\omega$ with $(\alpha, m) \in \operatorname{dom}(b)$,

$$
\left(p_{2}, q_{2}\right) \|-\underset{\sim}{\sim} \alpha(m)=\underset{\sim}{\sim} 2, f_{\alpha}\left(k_{\alpha}+m\right)(0)=p_{2}\left(2, f_{\alpha}\left(k_{\alpha}+m\right), 0\right)=b(\alpha, m)=b(\alpha)(m) .
$$

\footnotetext{
${ }^{5}$ Note that all the action in Cases 1-3 below is happening in the generic extension; in particular, we did not yet determine the value of $k_{\alpha}$.
} 
Thus $\left(p_{2}, q_{2}\right) \|-{ }^{*} \underset{\sim}{\sim} \alpha$ extend $b(\alpha)$ ", and hence

$$
\left(p_{2}, q_{2}\right) \|-"\left\langle\underset{\sim}{\sim} \alpha: \alpha \in J_{1} \cup J_{2}\right\rangle \text { extends }\left\langle b(\alpha): \alpha \in J_{1} \cup J_{2}\right\rangle " .
$$

By induction suppose that we have defined $\left(p_{1}, q_{1}\right) \geq\left(p_{2}, q_{2}\right) \geq \ldots \geq\left(p_{j}, q_{j}\right)$ for $j<t$, where for $1 \leq i \leq j$,

$$
\left(p_{i}, q_{i}\right) \|-"\left\langle\underset{\sim}{s} \alpha: \alpha \in J_{1} \cup \ldots \cup J_{i}\right\rangle \text { extends }\left\langle b(\alpha): \alpha \in J_{1} \cup \ldots \cup J_{i}\right\rangle " .
$$

We define $\left(p_{j+1}, q_{j+1}\right) \leq\left(p_{j}, q_{j}\right)$ such that for each $\alpha \in J_{j+1}$,

$$
\left(p_{j+1}, q_{j+1}\right) \|-“{ }_{\sim}^{s} \alpha \text { extends } b(\alpha) \text { ". }
$$

Let $\alpha \in J_{j+1}$. Then we can find $\eta \in T \uparrow j$ and $\alpha^{*}<\alpha^{* *}$ such that $\alpha^{*}, \alpha^{* *} \in$ $\operatorname{Suc}_{T}(\eta), \alpha^{* *}=\min \left(\operatorname{Suc}_{T}(\eta) \backslash\left(\alpha^{*}+1\right)\right)$ and $\alpha \in S u c_{T}\left(\eta \frown\left\langle\alpha^{* *}\right\rangle\right)$. As before there are three cases to be considered 6

Case 1. $\left|\alpha^{* *} \backslash \alpha^{*}\right| \leq \kappa$. Then let $i_{\alpha}$ be the index of $\alpha$ in the enumeration of $\alpha^{* *} \backslash \alpha^{*}$ considered in Case A and let $k_{\alpha}=\min \left\{m<\omega: r_{\pi\left(\eta-\left\langle\alpha^{* *}\right\rangle\right) \frown\left\langle i_{\alpha}\right\rangle}(m)=1\right\}$. Then

$$
\forall m<\omega, s_{\alpha}(m)=r_{j+1, f_{\alpha}\left(k_{\alpha}+m\right)}(0) .
$$

Case 2. $\left|\alpha^{* *} \backslash \alpha^{*}\right|>\kappa$ and $\rho=c f \alpha^{* *}<\kappa$. Let $\left\langle\alpha_{\nu}^{* *}: \nu<\rho\right\rangle$ be as in Case B and let $\nu_{\alpha}<\rho$ be such that $\alpha=\alpha_{\nu_{\alpha}}^{* *}$. Let $k_{\alpha}=\min \left\{m<\omega: r_{\pi\left(\eta-\left\langle\alpha^{* *}\right\rangle\right)}-\left\langle\nu_{\alpha}\right\rangle(m)=1\right\}$. Then

$$
\forall m<\omega, s_{\alpha}(m)=r_{j+1, f_{\alpha}\left(k_{\alpha}+m\right)}(0) .
$$

Case 3. $\rho=c f \alpha^{* *}>\kappa$. Let $\left\langle\alpha_{\nu}^{* *}: \nu<\rho\right\rangle$ be as in Case C. Let $\nu_{\alpha}<\rho$ be such that $\alpha=\alpha_{\nu_{\alpha}}^{* *}$, and let $k_{\alpha}=\min \left\{m<\omega: r_{\pi\left(\eta-\left\langle\alpha^{* *}\right\rangle\right) \frown\langle 0\rangle}(m)=1\right\}$. Then

$$
\forall m<\omega, s_{\alpha}(m)=r_{j+1, f_{\alpha}\left(k_{\alpha}+m\right)}(0) .
$$

Let $m^{*}<\omega$ be such that for all $n \geq m^{*}$ and $\alpha<\alpha^{\prime}$ in $J_{1} \cup \ldots \cup J_{j+1}, f_{\alpha}(n)<f_{\alpha^{\prime}}(n)$. Let

$$
\begin{aligned}
& q_{j+1}=q_{j} \cup\left\{\langle\bar{\eta}, n, 0\rangle: n<m^{*}, \exists \alpha \in J_{j+1} \text { (for some unique } \eta \in T \uparrow j\right. \text {, } \\
& \alpha^{* *} \in \operatorname{Suc}_{T}(\eta) \text {, we have } \alpha \in \operatorname{Suc}_{T}\left(\eta \frown\left\langle\alpha^{* *}\right\rangle\right) \\
& \text { and }\left(\bar{\eta}=\pi\left(\eta \frown\left\langle\alpha^{* *}\right\rangle\right) \frown\left\langle i_{\alpha}\right\rangle\right. \\
& \text { or } \bar{\eta}=\pi\left(\eta \frown\left\langle\alpha^{* *}\right\rangle\right) \frown\left\langle\nu_{\alpha}\right\rangle \\
& \text { or } \left.\left.\bar{\eta}=\left(\pi\left(\eta \frown\left\langle\alpha^{* *}\right\rangle\right) \frown\langle 0\rangle\right)\right)\right\} \\
& \cup\left\{\left\langle\bar{\eta}, m^{*}, 1\right\rangle: \exists \alpha \in J_{j+1} \text { (for some unique } \eta \in T\lceil j \text {, }\right. \\
& \alpha^{* *} \in \operatorname{Suc}_{T}(\eta) \text {, we have } \alpha \in \operatorname{Suc}_{T}\left(\eta \frown\left\langle\alpha^{* *}\right\rangle\right) \\
& \text { and }\left(\bar{\eta}=\pi\left(\eta \frown\left\langle\alpha^{* *}\right\rangle\right) \frown\left\langle i_{\alpha}\right\rangle\right. \\
& \text { or } \bar{\eta}=\pi\left(\eta \frown\left\langle\alpha^{* *}\right\rangle\right) \frown\left\langle\nu_{\alpha}\right\rangle \\
& \text { or } \left.\left.\bar{\eta}=\left(\pi\left(\eta \frown\left\langle\alpha^{* *}\right\rangle\right) \frown\langle 0\rangle\right)\right)\right\} \text {. }
\end{aligned}
$$

Let

It is easily seen that $q_{j+1} \in \mathbb{C}\left([\kappa]^{<\omega}\right)$, and for each $\alpha \in J_{j+1},\left(\phi, q_{j+1}\right) \|-k_{\alpha}=m^{*}$.

$$
p_{j+1}=p_{j} \cup\left\{\left\langle j+1, f_{\alpha}\left(m^{*}+m\right), 0, b(\alpha, m)\right\rangle: \alpha \in J_{j+1},(\alpha, m) \in \operatorname{dom}(b)\right\} .
$$

\footnotetext{
${ }^{6}$ Again note that all the action in Cases 1-3 below is happening in the generic extension.
} 
Then $p_{j+1} \in \mathbb{C}(\omega \times \kappa)$ is well defined and $\left(p_{j+1}, q_{j+1}\right) \leq\left(p_{j}, q_{j}\right)$, and for $\alpha \in J_{j+1}$ we have

$$
\begin{aligned}
\left(p_{j+1}, q_{j+1}\right) \|-\underbrace{s}_{\sim} \alpha & ={\underset{\sim}{\sim}}_{j+1, f_{\alpha}\left(k_{\alpha}+m\right)}(0)=p_{j+1}\left(j+1, f_{\alpha}\left(k_{\alpha}+m\right), 0\right) \\
& =b(\alpha, m)=b(\alpha)(m) .
\end{aligned}
$$

Thus $\left(p_{j+1}, q_{j+1}\right) \|-{ }^{\prime} \underset{\sim}{\sim} \alpha$ extends $b(\alpha)$ ". Finally let $(\bar{p}, \bar{q})=\left(p_{t}, q_{t}\right)$. Then for each component $\alpha$ of $b$,

$$
(\bar{p}, \bar{q}) \|-"{ }_{\sim} s_{\alpha} \text { extends } b(\alpha) "
$$

Hence

$$
(\bar{p}, \bar{q}) \|-"\langle\underset{\sim}{s} \alpha: \alpha \in I\rangle \text { extends } b " .
$$

(*) follows and we are done.

Theorem 2.1 follows.

We now give several applications of the above theorem.

Theorem 2.5. Suppose that $V$ satisfies $G C H, \kappa=\bigcup_{n<\omega} \kappa_{n}$ and $\bigcup_{n<\omega} o\left(\kappa_{n}\right)=\kappa$ (where o $\left(\kappa_{n}\right)$ is the Mitchell order of $\kappa_{n}$ ). Then there exists a cardinal preserving generic extension $V_{1}$ of $V$ satisfying $G C H$ and having the same reals as $V$ does, so that adding $\kappa$-many Cohen reals over $V_{1}$ produces $\kappa^{+}$-many Cohen reals over $V$.

Proof. Rearranging the sequence $\left\langle\kappa_{n}: n<\omega\right\rangle$ we may assume that $o\left(\kappa_{n+1}\right)>\kappa_{n}$ for each $n<\omega$. Let $0<n<\omega$. By Mag1, there exists a forcing notion $\mathbb{P}_{n}$ such that:

- Each condition in $\mathbb{P}_{n}$ is of the form $(g, G)$, where $g$ is an increasing function from a finite subset of $\kappa_{n}^{+}$into $\kappa_{n+1}$ and $G$ is a function from $\kappa_{n}^{+} \backslash \operatorname{dom}(g)$ into $\mathcal{P}\left(\kappa_{n+1}\right)$ such that for each $\alpha \in \operatorname{dom}(G), G(\alpha)$ belongs to a suitable normal measure 7 We may also assume that conditions have no parts below or at $\kappa_{n}$, and sets of measure one are like this as well.

- Forcing with $\mathbb{P}_{n}$ preserves cardinals and the $G C H$, and adds no new subsets to $\kappa_{n}$.

- If $G_{n}$ is $\mathbb{P}_{n}$-generic over $V$, then in $V\left[G_{n}\right]$ there is a normal function $g_{n}^{*}$ : $\kappa_{n}^{+} \longrightarrow \kappa_{n+1}$ such that $\operatorname{ran}\left(g_{n}^{*}\right)$ is a club subset of $\kappa_{n+1}$ consisting of measurable cardinals of $V$ such that $V\left[G_{n}\right]=V\left[g_{n}^{*}\right]$.

Let $\mathbb{P}^{*}=\prod_{n<\omega} \mathbb{P}_{n}$, and let

$$
\mathbb{P}=\left\{\left\langle\left\langle g_{n}, G_{n}\right\rangle: n<\omega\right\rangle \in \mathbb{P}^{*}: g_{n}=\emptyset \text {, for all but finitely many } n\right\} .
$$

Then $\mathbb{P}$ satisfies the $\kappa^{+}-$c.c. 8 and using simple modification of arguments from Mag1, Mag2 we can show that forcing with $\mathbb{P}$ preserves cardinals and the $G C H$. Let $G$ be $\mathbb{P}$-generic over $V$, and let $g_{n}^{*}: \kappa_{n}^{+} \longrightarrow \kappa_{n+1}$ be the generic function added by the part of the forcing corresponding to $\mathbb{P}_{n}$, for $0<n<\omega$. Let $X=$ $\bigcup_{0<n<\omega}\left(\left(\operatorname{ran}\left(g_{n}^{*}\right) \backslash \kappa_{n}^{+}\right) \cup\left\{\kappa_{n+1}\right\}\right)$ and let $g^{*}: \kappa \longrightarrow \kappa$ be an enumeration of $X$ in increasing order. Then $X=\operatorname{ran}\left(g^{*}\right)$ is club in $\kappa$ and consists entirely of measurable cardinals of $V$. Also, $V[G]=V\left[g^{*}\right]$.

\footnotetext{
${ }^{7}$ In fact, if $\alpha>\max (\operatorname{dom}(g))$, then $G(\alpha)$ belongs to a normal measure on $\kappa_{n+1}$, and if $\alpha<$ $\max (\operatorname{dom}(g))$, then $G(\alpha)$ belongs to a normal measure on $g(\beta)$ where $\beta=\min (\operatorname{dom}(g) \backslash \alpha)$.

${ }^{8}$ This is because any two conditions $\left\langle\left\langle g_{n}, G_{n}\right\rangle: n\langle\omega\rangle\right.$ and $\left\langle\left\langle g_{n}, H_{n}\right\rangle: n<\omega\right\rangle$ in $\mathbb{P}$ are compatible.
} 
Working in $V[G]$, let $\mathbb{Q}$ be the usual forcing notion for adding a club subset of $\kappa^{+}$which avoids points of countable $V$-cofinality. Thus $\mathbb{Q}=\{p: p$ is a closed bounded subset of $\kappa^{+}$and avoids points of countable $V$-cofinality\}, ordered by end extension. Let $H$ be $\mathbb{Q}$-generic over $V[G]$ and $C=\bigcup\{p: p \in H\}$.

Lemma 2.6. (a) $(\mathbb{Q}, \leq)$ satisfies the $\kappa^{++}$-c.c.,

(b) $(\mathbb{Q}, \leq)$ is $<\kappa^{+}$-distributive,

(c) $C$ is a club subset of $\kappa^{+}$which avoids points of countable $V$-cofinality.

$(a)$ and $(c)$ of the above lemma are trivial. For use later we prove a more general version of $(b)$.

Lemma 2.7. Let $V \subseteq W$, let $\nu$ be regular in $W$ and suppose that:

(a) $W$ is a $\nu$-c.c. extension of $V$.

(b) For every $\lambda<\nu$ which is regular in $W$, there is $\tau<\nu$ so that $c f^{W}(\tau)=\lambda$ and $\tau$ has a club subset in $W$ which avoids points of countable $V$-cofinality.

In $W$ let $\mathbb{Q}=\{p \subseteq \nu: p$ is closed and bounded in $\nu$ and avoids points of countable $V$-cofinality\}. Then in $W, \mathbb{Q}$ is $<\nu$-distributive.

Proof. This lemma first appeared in G-N-S. We prove it for completeness. Suppose that $W=V[G]$, where $G$ is $\mathbb{P}$-generic over $V$ for a $\nu$-c.c. forcing notion $\mathbb{P}$. Let $\lambda<\nu$ be regular, $q \in \mathbb{Q}, \underset{\sim}{f} \in W^{\mathbb{Q}}$ and

$$
q \|-\underset{\sim}{\sim}: \lambda \longrightarrow O N
$$

We find an extension of $q$ which decides $f$. By $(b)$ we can find $\tau<\nu$ and $g: \lambda \longrightarrow \tau$ such that $c f^{W}(\tau)=\lambda, g$ is normal and $C=\operatorname{ran}(g)$ is a club of $\tau$ which avoids points of countable $V$-cofinality.

In $W$, let $\theta>\nu$ be large enough regular. Working in $V$, let $\bar{H} \prec V_{\theta}$ and $R: \tau \longrightarrow O N$ be such that

- $\operatorname{Card}(\bar{H})<\nu$,

- $\bar{H}$ has $\lambda, \tau, \nu, \mathbb{P}$ and $\mathbb{P}$-names for $p, \mathbb{Q}, \underset{\sim}{f}, g$ and $C$ as elements,

- $\operatorname{ran}(R)$ is cofinal in $\sup (\bar{H} \cap \nu)$,

- $R \uparrow \beta \in \bar{H}$ for each $\beta<\tau$.

Let $H=\bar{H}[G]$. Then $\sup (H \cap \nu)=\sup (\bar{H} \cap \nu)$, since $\mathbb{P}$ is $\nu$-c.c., $H \prec V_{\theta}^{W}$, and if $\gamma=\sup (H \cap \nu)$, then $c f^{W}(\gamma)=c f^{W}(\tau)=\lambda$. For $\alpha<\lambda$ let $\gamma_{\alpha}=R(g(\alpha))$. Then

- $\left\langle\gamma_{\alpha}: \alpha<\lambda\right\rangle \in W$ is a normal sequence cofinal in $\gamma$,

- $\left\langle\gamma_{\alpha}: \alpha<\beta\right\rangle \in H$ for each $\beta<\lambda$, since $R \uparrow g(\beta) \in \bar{H}$,

- $c f^{V}\left(\gamma_{\alpha}\right)=c f^{V}(g(\alpha)) \neq \omega$ for each $\alpha<\lambda$, since $R$ is normal and $g(\alpha) \in C$.

Let $D=\left\{\gamma_{\alpha}: \alpha<\lambda\right\}$. We define by induction a sequence $\left\langle q_{\eta}: \eta<\lambda\right\rangle$ of conditions in $\mathbb{Q}$ such that for each $\eta<\lambda$,

- $q_{0}=q$,

- $q_{\eta} \in H$,

- $q_{\eta+1} \leq q_{\eta}$,

- $q_{\eta+1}$ decides $f(\eta)$

- $D \cap\left(\max \left(q_{\eta}\right) \stackrel{\sim}{,} \max \left(q_{\eta+1}\right)\right) \neq \emptyset$,

- $q_{\eta}=\bigcup_{\rho<\eta} q_{\rho} \cup\left\{\delta_{\eta}\right\}$, where $\delta_{\eta}=\sup _{\rho<\eta}\left(\max \left(q_{\rho}\right)\right)$, if $\eta$ is a limit ordinal.

We may further suppose that

- $q_{\eta}$ 's are chosen in a uniform way (say via a well-ordering which is built in to $\bar{H})$. 
We can define such a sequence using the fact that $H$ contains all initial segments of $D$ and that $\delta_{\eta} \in D$ for every limit ordinal $\eta<\lambda$ (and hence $c f^{V}\left(\delta_{\eta}\right) \neq \omega$ ).

Finally let $q_{\lambda}=\bigcup_{\eta<\lambda} q_{\eta} \cup\left\{\delta_{\lambda}\right\}$, where $\delta_{\lambda}=\sup _{\eta<\lambda}\left(\max \left(q_{\eta}\right)\right)$. Then $\delta_{\lambda} \in$ $D \cup\{\gamma\}$, hence $c f^{V}\left(\delta_{\lambda}\right) \neq \omega$. It follows that $q_{\lambda} \in \mathbb{Q}$ is well defined. Trivially $q_{\lambda} \leq q$ and $q_{\lambda}$ decides $\underset{\sim}{f}$. The lemma follows.

Let $V_{1}=V[G * H]$. The following is obvious:

Lemma 2.8. (a) $V$ and $V_{1}$ have the same cardinals and reals,

(b) $V_{1}=G C H$.

It follows from Theorem 2.1 that adding $\kappa$-many Cohen reals over $V_{1}$ adds $\kappa^{+}$many Cohen reals over $V$. This concludes the proof of Theorem 2.5.

Let us show that some large cardinals are needed for the previous result.

Theorem 2.9. Assume that $V_{1} \supseteq V$ and $V_{1}$ and $V$ have the same cardinals and reals. Suppose that for some uncountable cardinal $\kappa$ of $V_{1}$, adding $\kappa$-many Cohen reals to $V_{1}$ produces $\kappa^{+}$-many Cohen reals to $V$. Then in $V_{1}$ there is an inner model with a measurable cardinal.

Proof. Suppose on the contrary that in $V_{1}$ there is no inner model with a measurable cardinal. Thus by the Dodd-Jensen covering lemma (see D-J1, D-J2 $)\left(\mathcal{K}\left(V_{1}\right), V_{1}\right)$ satisfies the covering lemma, where $\mathcal{K}\left(V_{1}\right)$ is the Dodd-Jensen core model as computed in $V_{1}$.

Claim 2.10. $\mathcal{K}(V)=\mathcal{K}\left(V_{1}\right)$.

Proof. The claim is well known and follows from the fact that $V$ and $V_{1}$ have the same cardinals. We present a proof for completeness 9 Suppose not. Clearly $\mathcal{K}(V) \subseteq \mathcal{K}\left(V_{1}\right)$, so let $A \subseteq \alpha, A \in \mathcal{K}\left(V_{1}\right), A \notin \mathcal{K}(V)$. Then there is a mouse of $\mathcal{K}\left(V_{1}\right)$ to which $A$ belongs, hence there is such a mouse of $\mathcal{K}\left(V_{1}\right)$-power $\alpha$. It then follows that for every limit cardinal $\lambda>\alpha$ of $V_{1}$ there is a mouse with critical point $\lambda$ to which $A$ belongs, and the filter is generated by end segments of

$$
\left\{\chi: \chi<\lambda, \chi \text { a cardinal in } V_{1}\right\} .
$$

As $V$ and $V_{1}$ have the same cardinals, this mouse is in $V$, and hence in $\mathcal{K}(V)$.

Let us denote this common core model by $\mathcal{K}$. Then $\mathcal{K} \subseteq V$, and hence $\left(V, V_{1}\right)$ satisfies the covering lemma. It follows that $\left(\left[\kappa^{+}\right] \leq \omega_{1}\right)^{V}$ is unbounded in $\left(\left[\kappa^{+}\right] \leq \omega\right)^{V_{1}}$, and since $\omega_{1}^{V}=\omega_{1}^{V_{1}}$, we can easily show that $\left(\left[\kappa^{+}\right]^{\leq \omega}\right)^{V}$ is unbounded in $\left(\left[\kappa^{+}\right]^{\leq \omega}\right)^{V_{1}}$. Since $V_{1}$ and $V$ have the same reals, $\left(\left[\kappa^{+}\right]^{\leq \omega}\right)^{V}=\left(\left[\kappa^{+}\right]^{\leq \omega}\right)^{V_{1}}$, and we get a contradiction.

If we relax our assumptions, and allow some cardinals to collapse, then no large cardinal assumptions are needed.

Theorem 2.11. (a) Suppose $V$ is a model of GCH. Then there is a generic extension $V_{1}$ of $V$ satisfying $G C H$ so that the only cardinal of $V$ which is collapsed in $V_{1}$ is $\aleph_{1}$ and such that adding $\aleph_{\omega}$-many Cohen reals to $V_{1}$ produces $\aleph_{\omega+1}$-many of them over $V$.

(b) Suppose $V$ satisfies $G C H$. Then there is a generic extension $V_{1}$ of $V$ satisfying $G C H$ and having the same reals as $V$ does, so that the only cardinals of $V$

\footnotetext{
${ }^{9}$ Our proof is the same as in the proof of [Sh2, Theorem VII. 4.2(1)].
} 
which are collapsed in $V_{1}$ are $\aleph_{2}$ and $\aleph_{3}$ and such that adding $\aleph_{\omega}$-many Cohen reals to $V_{1}$ produces $\aleph_{\omega+1}$-many of them over $V$.

Proof. (a) Working in $V$, let $\mathbb{P}=\operatorname{Col}\left(\aleph_{0}, \aleph_{1}\right)$ and let $G$ be $\mathbb{P}$-generic over $V$. Also let $S=\left\{\alpha<\omega_{2}: c f^{V}(\alpha)=\omega_{1}\right\}$. Then $S$ remains stationary in $V[G]$. Working in $V[G]$, let $\mathbb{Q}$ be the standard forcing notion for adding a club subset of $S$ with countable conditions, and let $H$ be $\mathbb{Q}$-generic over $V[G]$. Let $C=\bigcup H$. Then $C$ is a club subset of $\omega_{1}^{V[G]}=\omega_{2}^{V}$ such that $C \subseteq S$, and in particular $C$ avoids points of countable $V$-cofinality. Working in $V[G * H]$, let

$$
\mathbb{R}=\left\langle\left\langle\mathbb{P}_{\nu}: \aleph_{2} \leq \nu \leq \aleph_{\omega+2}, \nu \text { regular }\right\rangle,\left\langle\underset{\sim}{\mathbb{Q}} \nu: \aleph_{2} \leq \nu \leq \aleph_{\omega+1}, \nu \text { regular }\right\rangle\right\rangle
$$

be the Easton support iteration by letting $\mathbb{Q}_{\nu}$ name the poset $\{p \subset \nu: p$ is closed and bounded in $\nu$ and avoids points of countable $V$-cofinality $\}$ as defined in $V[G * H]^{\mathbb{P}_{\nu}}$. Let

$$
K=\left\langle\left\langle G_{\nu}: \aleph_{2} \leq \nu \leq \aleph_{\omega+2}, \nu \text { regular }\right\rangle,\left\langle H_{\nu}: \aleph_{2} \leq \nu \leq \aleph_{\omega+1}, \nu \text { regular }\right\rangle\right\rangle
$$

be $\mathbb{R}$-generic over $V[G * H]$ (i.e. $G_{\nu}$ is $\mathbb{P}_{\nu}$-generic over $V[G * H]$ and $H_{\nu}$ is $\mathbb{Q}_{\nu}=$ $\mathbb{Q}_{\nu}\left[G_{\nu}\right]$-generic over $\left.V\left[G * H * G_{\nu}\right]\right)$. Then

Lemma 2.12. (a) $\mathbb{P}_{\nu}$ adds a club disjoint from $\left\{\alpha<\lambda: c f^{V}(\alpha)=\omega\right\}$ for each regular $\lambda \in\left(\aleph_{1}, \nu\right)$.

(b) (By Lemma 2.7) $V\left[G * H * G_{\nu}\right] \models$ "Q্Q ${ }_{\nu}$ is $<\nu$-distributive".

(c) $V[G * H]$ and $V[G * H * K]$ have the same cardinals and reals, and satisfy $G C H$.

(d) In $V[G * H * K]$ there is a club subset $C$ of $\aleph_{\omega+1}$ which avoids points of countable $V$-cofinality.

Let $V_{1}=V[G * H * K]$. By the above results, $V_{1}$ satisfies $G C H$ and the only cardinal of $V$ which is collapsed in $V_{1}$ is $\aleph_{1}$. The proof of the fact that adding $\aleph_{\omega}$-many Cohen reals over $V_{1}$ produces $\aleph_{\omega+1}$-many of them over $V$ follows from Theorem 2.1.

(b) Working in $V$, let $\mathbb{P}$ be the following version of Namba forcing:

$\mathbb{P}=\left\{T \subseteq \omega_{2}^{<\omega}: T\right.$ is a tree, and for every $s \in T$,

the set $\{t \in T: t \supset s\}$ has size $\left.\aleph_{2}\right\}$

ordered by inclusion. Let $G$ be $\mathbb{P}$-generic over $V$. It is well known that forcing with $\mathbb{P}$ adds no new reals, preserves cardinals $\geq \aleph_{4}$ and that $\left|\aleph_{2}^{V}\right|^{V[G]}=\left|\aleph_{3}^{V}\right|^{V[G]}=$ $\aleph_{1}^{V[G]}=\aleph_{1}^{V}$ (see [Sh1]). Let $S=\left\{\alpha<\omega_{3}: c f^{V}(\alpha)=\omega_{2}\right\}$.

Lemma 2.13. $S$ remains stationary in $V[G]$.

Proof. See [Ve-W, Lemma 3].

Now the rest of the proof is exactly as in $(a)$.

The theorem follows.

By the same line but using stronger initial assumptions, adding $\kappa$-many Cohen reals may produce $\lambda$-many of them for $\lambda$ much larger than $\kappa^{+}$.

Theorem 2.14. Suppose that $\kappa$ is a strong cardinal, $\lambda \geq \kappa$ is regular and $G C H$ holds. Then there exists a cardinal preserving generic extension $V_{1}$ of $V$ having the same reals as $V$ does, so that adding $\kappa$-many Cohen reals over $V_{1}$ produces $\lambda$-many of them over $V$. 
Proof. Working in $V$, build for each $\delta$ a measure sequence $\vec{u}_{\delta}$ from a $j$ witnessing " $\kappa$ is $\delta$-strong" out to the first weak repeat point. Find $\vec{u}$ such that $\vec{u}=\vec{u}_{\delta}$ for unboundedly many $\delta$. Let $\mathbb{R}_{\vec{u}}$ be the corresponding Radin forcing notion and let $G$ be $\mathbb{R}_{\vec{u}}$-generic over $V$. Then

Lemma 2.15. (a) Forcing with $\mathbb{R}_{\vec{u}}$ preserves cardinals and the $G C H$ and adds no new reals.

(b) In $V[G]$, there is a club $C_{\kappa} \subseteq \kappa$ consisting of inaccessible cardinals of $V$ and $V[G]=V\left[C_{\kappa}\right]$.

(c) $\kappa$ remains strong in $V[G]$.

Proof. See Git2] and $\mathrm{Cu}$.

Working in $V[G]$, let

$$
E=\left\langle\left\langle U_{\alpha}: \alpha<\lambda\right\rangle,\left\langle\pi_{\alpha \beta}: \alpha \leq_{E} \beta\right\rangle\right\rangle
$$

be a nice system satisfying conditions (0)-(9) in [Git2, page 37]. Also let

$$
\mathbb{R}=\left\langle\left\langle\mathbb{P}_{\nu}: \kappa^{+} \leq \nu \leq \lambda^{+}, \nu \text { regular }\right\rangle,\left\langle\underset{\sim}{\mathbb{Q}}: \kappa^{+} \leq \nu \leq \lambda, \nu \text { regular }\right\rangle\right\rangle
$$

be the Easton support iteration by letting $\mathbb{Q}_{\nu}$ name the poset $\{p \subseteq \nu: p$ is closed and bounded in $\nu$ and avoids points of countable $V$-cofinality $\}$ as defined in $V[G]^{\mathbb{P}_{\nu}}$. Let

$$
K=\left\langle\left\langle G_{\nu}: \kappa^{+} \leq \nu \leq \lambda^{+}, \nu \text { regular }\right\rangle,\left\langle H_{\nu}: \kappa^{+} \leq \nu \leq \lambda, \nu \text { regular }\right\rangle\right\rangle
$$

be $\mathbb{R}$-generic over $V[G]$. Then

Lemma 2.16. (a) $\mathbb{P}_{\nu}$ adds a club disjoint form $\left\{\alpha<\delta: c f^{V}(\alpha)=\omega\right\}$ for each regular $\delta \in(\kappa, \nu)$.

(b) (By Lemma 2.7) $V\left[G * G_{\nu}\right] \models " \mathbb{Q}_{\nu}=\mathbb{Q}_{\nu}\left[G_{\nu}\right]$ is < $<$-distributive".

(c) $V[G]$ and $V[G * K]$ have the same cardinals, and satisfy $G C H$.

(d) $\mathbb{R}$ is $\leq \kappa$-distributive, hence forcing with $\mathbb{R}$ adds no new $\kappa$-sequences.

(e) In $V[G * K]$, for each regular cardinal $\kappa \leq \nu \leq \lambda$ there is a club $C_{\nu} \subseteq \nu$ such that $C_{\nu}$ avoids points of countable $V$-cofinality.

By Lemma $2.16(d), E$ remains a nice system in $V[G * K]$, except that the condition $(0)$ is replaced by $\left(\lambda, \leq_{E}\right)$ is $\kappa^{+}$-directed closed. Hence working in $V[G * K]$, by results of Git-Mag1, Git-Mag2 and Mer, we can find a forcing notion $S$ such that if $L$ is $S$-generic over $V[G * K]$, then

- $V[G * K]$ and $V[G * K * L]$ have the same cardinals and reals.

- In $V[G * K * L], 2^{\kappa}=\lambda, c f(\kappa)=\aleph_{0}$ and there is an increasing sequence $\left\langle\kappa_{n}: n<\omega\right\rangle$ of regular cardinals cofinal in $\kappa$ and an increasing (mod finite) sequence $\left\langle f_{\alpha}: \alpha<\lambda\right\rangle$ in $\prod_{n<\omega}\left(\kappa_{n+1} \backslash \kappa_{n}\right)$.

Let $V_{1}=V[G * K * L]$. Then $V_{1}$ and $V$ have the same cardinals and reals. The fact that adding $\kappa$-many Cohen reals over $V_{1}$ produces $\lambda$-many Cohen reals over $V$ follows from Theorem 2.1. 
If we allow many cardinals between $V$ and $V_{1}$ to collapse, then using Git-Mag1, Sec. 2] one can obtain the following:

Theorem 2.17. Suppose that there is a strong cardinal and GCH holds. Let $\alpha<\omega_{1}$. Then there is a model $V_{1} \supset V$ having the same reals as $V$ and satisfying GCH below $\aleph_{\omega}^{V_{1}}$ such that adding $\aleph_{\omega}^{V_{1}}$-many Cohen reals to $V_{1}$ produces $\aleph_{\alpha+1}^{V_{1}}$-many of them over $V$.

Proof. Proceed as in Theorem 2.14 to produce the model $V[G * K]$. Then working in $V[G * K]$, we can find a forcing notion $S$ such that if $L$ is $S$-generic over $V[G * K]$, then

- $V[G * K]$ and $V[G * K * L]$ have the same reals.

- In $V[G * K * L]$, cardinals $\geq \kappa$ are preserved, $\kappa=\aleph_{\omega}, G C H$ holds below $\aleph_{\omega}, 2^{\kappa}=\aleph_{\alpha+1}$ and there is an increasing (mod finite) sequence $\left\langle f_{\beta}: \beta<\right.$ $\left.\aleph_{\alpha+1}\right\rangle$ in $\prod_{n<\omega}\left(\aleph_{n+1} \backslash \aleph_{n}\right)$.

Let $V_{1}=V[G * K * L]$. Then $V_{1}$ and $V$ have the same reals. The fact that adding $\aleph_{\omega}^{V_{1}}$-many Cohen reals over $V_{1}$ produces $\aleph_{\alpha+1}^{V_{1}}$-many Cohen reals over $V$ follows from Theorem 2.1.

\section{Models with the Same Cofinality Function But Different ReAls}

This section is completely devoted to the proof of the following theorem.

Theorem 3.1. Suppose that $V$ satisfies $G C H$. Then there is a cofinality preserving generic extension $V_{1}$ of $V$ satisfying $G C H$ so that adding a Cohen real over $V_{1}$ produces $\aleph_{1}$-many Cohen reals over $V$.

The basic idea of the proof will be to split $\omega_{1}$ into $\omega$ sets such that none of them will contain an infinite set of $V$. Then something like in section 2 will be used for producing Cohen reals. It turned out however that just not containing an infinite set of $V$ is not enough. We will use a stronger property. As a result the forcing turns out to be more complicated. We are now going to define the forcing sufficient for proving the theorem. Fix a nonprincipal ultrafilter $U$ over $\omega$.

Definition 3.2. Let $\left(\mathbb{P}_{U}, \leq, \leq^{*}\right)$ be the Prikry (or in this context Mathias) forcing with $U$, i.e.

- $\mathbb{P}_{U}=\left\{\langle s, A\rangle \in[\omega]^{<\omega} \times U: \max (s)<\min (A)\right\}$,

- $\langle t, B\rangle \leq\langle s, A\rangle \Longleftrightarrow t$ end extends $s$ and $(t \backslash s) \cup B \subseteq A$,

- $\langle t, B\rangle \leq^{*}\langle s, A\rangle \Longleftrightarrow t=s$ and $B \subseteq A$.

We call $\leq *$ a direct or $*$-extension. The following are the basic facts on this forcing that will be used further.

Lemma 3.3. (a) The generic object of $\mathbb{P}_{U}$ is generated by a real.

(b) $\left(\mathbb{P}_{U}, \leq\right)$ satisfies the c.c.c.

(c) If $\langle s, A\rangle \in \mathbb{P}_{U}$ and $b \subseteq \omega \backslash(\max (s)+1)$ is finite, then there is a $*$-extension of $\langle s, A\rangle$, forcing the generic real to be disjoint to $b$.

Proof. (a) If $G$ is $\mathbb{P}_{U}$-generic over $V$, then let $r=\bigcup\{s: \exists A,\langle s, A\rangle \in G\}$. $r$ is a real and $G=\left\{\langle s, A\rangle \in \mathbb{P}_{U}: r\right.$ end extends $s$ and $\left.r \backslash s \subseteq A\right\}$.

(b) Trivial using the fact that for $\langle s, A\rangle,\langle t, B\rangle \in \mathbb{P}_{U}$, if $s=t$, then $\langle s, A\rangle$ and $\langle t, B\rangle$ are compatible.

(c) Consider $\langle s, A \backslash(\max (b)+1)\rangle$. 
We now define our main forcing notion.

Definition 3.4. $p \in \mathbb{P}$ iff $p=\left\langle p_{0}, \underset{\sim}{p_{1}}\right\rangle$ where

(1) $p_{0} \in \mathbb{P}_{U}$

(2) $\underset{\sim}{p_{1}}$ is a $\mathbb{P}_{U}$-name such that for some $\alpha<\omega_{1}, p_{0} \|-{\underset{\sim}{p}}_{1}: \alpha \longrightarrow \omega$ and such that the following hold

(2a) For every $\beta<\alpha, \underset{\sim}{p}{ }_{1}(\beta) \subseteq \mathbb{P}_{U} \times \omega$ is a $\mathbb{P}_{U}$-name for a natural number such that

- $p_{1}(\beta)$ is partial function from $\mathbb{P}_{U}$ into $\omega$,

- for some fixed $l<\omega$, $\operatorname{dom} p_{1}(\beta) \subseteq\left\{\langle s, \omega \backslash \max (s)+1\rangle: s \in[\omega]^{l}\right\}$,

- for all $\beta_{1} \neq \beta_{2}<\alpha$, range $\left({\underset{\sim}{\sim}}_{1}\left(\beta_{1}\right)\right) \cap \operatorname{range}\left({\underset{\sim}{p}}_{1}\left(\beta_{2}\right)\right)$ is finite 10

(2b) for every $I \subseteq \alpha, I \in V, p_{0}^{\prime} \stackrel{\sim}{\leq} p_{0}$ and finite $\widetilde{J \subseteq \omega}$ there is a finite set $a \subseteq \alpha$ such that for every finite set $b \subseteq I \backslash a$ there is $p_{0}^{\prime \prime} \leq^{*} p_{0}^{\prime}$ such that $p_{0}^{\prime \prime} \|-\left(\forall \beta \in b, \forall k \in J, \underset{\sim}{p}{ }_{1}(\beta) \neq k\right) \&\left(\forall \beta_{1} \neq \beta_{2} \in b, \underset{\sim}{p}{ }_{1}\left(\beta_{1}\right) \neq \underset{\sim}{p} p_{1}\left(\beta_{2}\right)\right)$.

Notation 3.5. (1) Call $\alpha$ the length of $p\left(\right.$ or $\left.p_{1}\right)$ and denote it by $\operatorname{lh}(p)\left(\operatorname{or} \operatorname{lh}\left({\underset{\sim}{\sim}}_{1}\right)\right)$.

(2) For $n<\omega$ let $\underset{\sim}{I} p, n$ be a $\mathbb{P}_{U}$-name such that $p_{0} \|-\underset{\sim}{\sim} p, n=\left\{\beta<\alpha:{\underset{\sim}{\sim}}_{1}(\beta) \stackrel{\sim}{\sim} n\right\}$. Then we can identify $\underset{\sim}{p}$ with $\langle\underset{\sim}{\underset{I}{I} p, n}: n<\omega\rangle$.

Remark 3.6. (2a) will guarantee that for $\beta<\alpha, p_{0} \|-p_{1}(\beta) \in \omega$. The last condition in $(2 \mathrm{a})$ is a technical fact that will be used in several parts of the argument. The condition (2b) appears technical but will be crucial for producing numerous Cohen reals.

Definition 3.7. For $p=\left\langle p_{0}, \underset{\sim}{p_{1}}\right\rangle, q=\left\langle q_{0}, \underset{\sim}{q_{1}}\right\rangle \in \mathbb{P}$, define

(1) $p \leq q$ iff

- $p_{0} \leq_{\mathbb{P}_{U}} q_{0}$,

- $\operatorname{lh}(q) \leq \operatorname{lh}(p)$

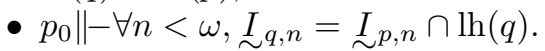

(2) $p \leq^{*} q$ iff

- $p_{0} \leq_{\mathbb{P}_{U}}^{*} q_{0}$,

- $p \leq q$.

We call $\leq^{*}$ a direct or $*$-extension.

Remark 3.8. In the definition of $p \leq q$, we can replace the last condition by $p_{0} \|-{\underset{\sim}{q}}_{1}=\underset{\sim}{p_{1}}\lceil\operatorname{lh}(q)$.

Lemma 3.9. Let $\left\langle p_{0}, \underset{*}{p_{1}}\right\rangle \|-$ " $\alpha$ is an ordinal". Then there are $\mathbb{P}_{U}$-names $\underset{\sim}{\beta}$ and ${\underset{\sim}{q}}_{1}$ such that $\left\langle p_{0}, \underset{\sim}{q_{1}}\right\rangle \widetilde{\Sigma}^{*}\left\langle p_{0}, \underset{\sim}{p_{1}}\right\rangle$ and $\left\langle p_{0}, \underset{\sim}{q_{1}}\right\rangle \|-\underset{\sim}{\alpha}=\underset{\sim}{\beta}$.

Proof. Suppose for simplicity that $\left\langle p_{0}, p_{1}\right\rangle=\langle\langle\langle\rangle, \omega\rangle, \phi\rangle$. Let $\theta$ be large enough regular and let $\left\langle N_{n}: n<\omega\right\rangle$ be an increasing sequence of countable elementary submodels of $H_{\theta}$ such that $\mathbb{P}, \underset{\sim}{\alpha} \in N_{0}$ and $N_{n} \in N_{n+1}$ for each $n<\omega$. Let $N=\bigcup_{n<\omega} N_{n}, \delta_{n}=N_{n} \cap \omega_{1}$ for $n<\omega$ and $\delta=\bigcup_{n<\omega} \delta_{n}=N \cap \omega_{1}$. Let $\left\langle J_{n}: n<\right.$ $\omega\rangle \in N_{0}$ be a sequence of infinite subsets of $\omega \backslash\{0\}$ such that $\bigcup_{n<\omega} J_{n}=\omega \backslash\{0\}$, $J_{n} \subseteq J_{n+1}$, and $J_{n+1} \backslash J_{n}$ is infinite for each $n<\omega$. Also let $\left\langle\alpha_{i}: 0<i<\omega\right\rangle$ be an enumeration of $\delta$ such that for every $n<\omega,\left\{\alpha_{i}: i \in J_{n}\right\} \in N_{n+1}$ is an enumeration of $\delta_{n}$ and $\left\{\alpha_{i}: i \in J_{n+1}\right\} \cap \delta_{n}=\left\{\alpha_{i}: i \in J_{n}\right\}$.

\footnotetext{
${ }^{10}$ Thus if $G$ and $r$ are as in the proof of Lemma 3.3 with $p_{0} \in G$, then $p_{o} \|-$ " ${\underset{\sim}{1}}_{1}(\beta)$ is the $l$-th element of $r "$
} 
We define by induction on the length of $s$, a sequence $\left\langle p^{s}: s \in[\omega]^{<\omega}\right\rangle$ of conditions such that

- $p^{s}=\left\langle p_{0}^{s}, \underset{\sim}{p}{ }_{1}^{s}\right\rangle=\left\langle\left\langle s, A_{s}\right\rangle, \underset{\sim}{p}\right\rangle$,

- $p^{s} \in N_{s(\ln (s)-1)+1}$,

- $\operatorname{lh}\left(p^{s}\right)=\delta_{s(\operatorname{lh}(s)-1)+1}$,

- if $t$ does not contradict $p_{0}^{s}$ (i.e. if the $t$ end extends $s$ and $t \backslash s \subseteq A_{S}$ ), then $p^{t} \leq p^{s}$.

For $s=\langle\rangle$, let $p^{\langle\rangle}=\langle\langle\langle\rangle, \omega\rangle, \phi\rangle$. Suppose that \langle\rangle$\neq s \in[\omega]^{<\omega}$ and $p^{s \mid \ln (s)-1}$ is defined. We define $p^{s}$. First we define $t^{s \mid \operatorname{lh}(s)-1} \leq^{*} p^{s \uparrow \operatorname{lh}(s)-1}$ as follows: If there is no $*$-extension of $p^{s \mid \ln (s)-1}$ deciding $\underset{\sim}{\alpha}$, then let $t^{s \mid \ln (s)-1}=p^{s \uparrow \ln (s)-1}$. Otherwise let $t^{s \uparrow \ln (s)-1} \in N_{s(\operatorname{lh}(s)-2)+1}$ be such an extension. Note that $\operatorname{lh}\left(t^{s \mid \ln (s)-1}\right) \leq$ $\delta_{s(\ln (s)-2)+1}$.

Let $t^{s\lceil\operatorname{lh}(s)-1}=\left\langle t_{0}, \underset{\sim}{\sim} 1\right\rangle, t_{0}=\langle s\lceil\operatorname{lh}(s)-1, A\rangle$. Let $C \subseteq \omega$ be an infinite set almost disjoint to $\langle$ range $(\underset{\sim}{\sim} 1(\beta)): \beta<\ln (\underset{\sim}{\underset{\sim}{t}} 1)\rangle$. Split $C$ into $\omega$ infinite disjoint sets $C_{i}, i<\omega$. Let $\left\langle c_{i j}: j<\omega\right\rangle$ be an increasing enumeration of $C_{i}, i<\omega$. We may suppose that all of these is done in $N_{s(\operatorname{lh}(s)-1)+1}$. Let $p^{s}=\left\langle p_{0}^{s}, \underset{\sim}{p_{1}^{s}}\right\rangle$, where

- $p_{0}^{s}=\langle s, A \backslash(\max (s)+1)\rangle$,

- for $\beta<\operatorname{lh}(\underset{\sim}{t} 1), p_{1}^{s}(\beta)=\underset{\sim}{t} 1(\beta)$,

- for $i \in J_{s(\ln (s)-1)}$ such that $\alpha_{i} \in \delta_{s(\ln (s)-1)} \backslash \ln (\underset{\sim}{\sim} 1)$,

$\underset{\sim}{p}{ }_{1}^{s}\left(\alpha_{i}\right)=\left\{\left\langle\left\langle s \frown\left\langle r_{1}, \ldots, r_{i}\right\rangle, \omega \backslash\left(r_{i}+1\right)\right\rangle, c_{i r_{i}}\right\rangle: r_{1}>\max (s),\left\langle r_{1}, \ldots, r_{i}\right\rangle \in[\omega]^{i}\right\}$.

Trivially $p^{s} \in N_{s(\operatorname{lh}(s)-1)+1}, \operatorname{lh}\left(p^{s}\right)=\delta_{s(\operatorname{lh}(s)-1)}$, and if $s(\operatorname{lh}(s)-1) \in A$, then $p^{s} \leq t^{s \mid \ln (s)-1}$.

Claim 3.10. $p^{s} \in \mathbb{P}$.

Proof. We check the conditions in Definition 3.4.

(1) i.e. $p_{0}^{s} \in \mathbb{P}_{U}$ is trivial.

(2) It is clear that $p_{0}^{s} \|-p_{1}^{s}: \delta_{s(\mathrm{hh}(s)-1)} \longrightarrow \omega$ and that (2a) holds. Let us prove (2b). Thus suppose that $I \subseteq \delta_{s(\mathrm{~h}(s)-1)}, I \in V, p \leq p_{0}^{s}$ and $J \subseteq \omega$ is finite. First we apply $(2 \mathrm{~b})$ to $\langle p, \underset{\sim}{\sim} 1\rangle, I \cap \operatorname{lh}(\underset{\sim}{\sim} 1), p$ and $J$ to find a finite set $a^{\prime} \subseteq \operatorname{lh}(\underset{\sim}{\sim} 1)$ such that

For every finite set $b \subseteq I \cap \ln \left(\underset{\sim}{t_{1}}\right) \backslash a^{\prime}$ there is $p^{\prime} \leq^{*} p$ such that $p^{\prime} \|-(\forall \beta \in \bar{b}, \forall k \in \widetilde{J, \underset{\sim}{t}} 1(\beta) \neq k) \&\left(\forall \beta_{1} \neq\right.$ $\left.\beta_{2} \in b, \underset{\sim}{t} 1\left(\beta_{1}\right) \neq \underset{\sim}{t} \underset{1}{\sim}\left(\beta_{2}\right)\right)$.

Let $p=\left\langle s \frown\left\langle r_{1}, \ldots, r_{m}\right\rangle, B\right\rangle$. Suppose that $\delta_{s(\ln (s)-1)} \backslash \operatorname{lh}(\underset{\sim}{t} 1)=\left\{\alpha_{J_{1}}, \ldots, \alpha_{J_{i}}, \ldots\right\}$, where $J_{1}<J_{2}<\ldots$ are in $J_{s(\operatorname{lh}(s)-1)}$. Let

$$
a=a^{\prime} \cup\left\{\alpha_{J_{1}}, \ldots, \alpha_{J_{m}}\right\} .
$$

We show that $a$ is as required. Thus suppose that $b \subseteq I \backslash a$ is finite. Apply (*) to $b \cap \operatorname{lh}\left(\underset{\sim}{t_{1}}\right)$ to find $p^{\prime}=\left\langle s^{\frown}\left\langle r_{1}, \ldots, r_{m}\right\rangle, B^{\prime}\right\rangle \leq^{*} p$ such that

$$
\begin{aligned}
& p^{\prime} \|-\left(\forall \beta \in b \cap \operatorname{lh}(\underset{\sim}{\sim} 1), \forall k \in J, \underset{\sim}{t}{ }_{1}(\beta) \neq k\right) \\
& \&\left(\forall \beta_{1} \neq \beta_{2} \in b \cap \operatorname{lh}(\underset{\sim}{\sim} 1), \underset{\sim}{t} 1\left(\beta_{1}\right) \neq \underset{\sim}{t} 1\left(\beta_{2}\right)\right) .
\end{aligned}
$$

Also note that

$$
p^{\prime} \|-\forall \beta \in b \cap \operatorname{lh}(\underset{\sim}{t} 1), \underset{\sim}{p}{ }_{1}^{s}(\beta)=\underset{\sim}{t} 1(\beta) .
$$


Pick $k<\omega$ such that

$\forall \beta \in b \cap \operatorname{lh}(\underset{\sim}{t} 1), \forall \alpha_{i} \in b \backslash \operatorname{lh}(\underset{\sim}{t} 1), \operatorname{range}\left({\underset{\sim}{p}}_{1}^{s}\left(\beta_{1}\right)\right) \cap\left(\operatorname{range}\left({\underset{\sim}{p}}_{1}^{s}\left(\alpha_{i}\right)\right) \backslash k\right)=\phi$.

Let $q=\left\langle s \frown\left\langle r_{1}, \ldots, r_{m}\right\rangle, B\right\rangle=\left\langle s \frown\left\langle r_{1}, \ldots, r_{m}\right\rangle, B^{\prime} \backslash(\max (J)+k+1)\right\rangle$. Then $q \leq^{*} p^{\prime} \leq^{*} p$. We show that $q$ is as required. We need to show that

(1) $q \|-\forall \beta \in b \backslash \operatorname{lh}\left(\underset{\sim}{t_{1}}\right), \forall k \in J, p_{1}^{s}(\beta) \neq k$,

(2) $q \|-\forall \beta_{1} \neq \beta_{2} \in \widetilde{b} \backslash \ln (\underset{\sim}{t} 1), \underset{p_{1}^{s}}{\widetilde{r}}\left(\beta_{1}\right) \neq p_{1}^{s}\left(\beta_{2}\right)$,

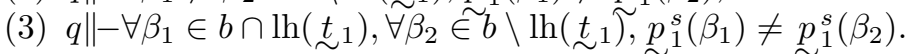

Now (1) follows from the fact that $q \|-" p_{1}^{s}\left(\alpha_{i}\right) \geq(i-m)$-th element of $B>$ $\max (J) "$. (2) follows from the fact that for $i \neq j<\omega, C_{i} \cap C_{j}=\emptyset$, and range $\left({\underset{\sim}{p}}_{1}^{s}\left(\alpha_{i}\right)\right) \subseteq C_{i}$. (3) follows from the choice of $k$. The claim follows.

This completes our definition of the sequence $\left\langle p^{s}: s \in[\omega]^{<\omega}\right\rangle$. Let

$$
\underset{\sim}{q} 1=\left\{\left\langle p_{0}^{s},\left\langle\beta, \underset{\sim}{p} p_{1}^{s}(\beta)\right\rangle\right\rangle: s \in[\omega]^{<\omega}, \beta<\operatorname{lh}\left(p^{s}\right)\right\} .
$$

Then $\underset{\sim}{q} 1$ is a $\mathbb{P}_{U}$-name, and for $s \in[\omega]<\omega, p_{0}^{s} \|-{\underset{\sim}{p}}_{1}^{s}={\underset{\sim}{q}}_{1}\left\lceil\operatorname{lh}\left({\underset{\sim}{p}}_{1}^{s}\right)\right.$.

Claim 3.11. $\langle\langle\langle\rangle, \omega\rangle, \underset{\sim}{q} 1\rangle \in \mathbb{P}$.

Proof. We check conditions in Definition 3.4.

(1) i.e. $\langle\langle\rangle, \omega\rangle \in \mathbb{P}_{U}$ is trivial.

(2) It is clear from our definition that

$$
\langle\langle\rangle, \omega\rangle \|-\stackrel{\sim}{\sim} q_{1} \text { is a well-defined function into } \omega " .
$$

Let us show that $\ln \left(\sim_{1}\right)=\delta$. By the construction it is trivial that $\ln \left({\underset{\sim}{q}}_{1}\right) \leq \delta$. We show that $\operatorname{lh}\left({\underset{\sim}{q}}_{1}\right) \geq \widetilde{\delta}$. It suffices to prove the following:

(*) For every $\tau<\delta$ and $p \in \mathbb{P}_{U}$ there is $q \leq p$ such that $q \|-{ }_{\sim} q_{1}(\tau)$ is defined".

Fix $\tau<\delta$ and $p=\langle s, A\rangle \in \mathbb{P}_{U}$ as in (*). Let $t$ be an end extension of $s$ such that $t \backslash s \subseteq A$ and $\delta_{t(\operatorname{lh}(t)-1)}>\tau$. Then $p_{0}^{t}$ and $p$ are compatible and $p_{0}^{t} \|-"{ }_{\sim}^{q} 1(\tau)={\underset{\sim}{p}}_{1}^{t}(\tau)$ is defined". Let $q \leq p_{0}^{t}, p$. Then $q \|-$ " $q_{1}(\tau)$ is defined" and $(*)$ follows. Thus $\ln \left({\underset{\sim}{q}}_{1}\right)=\delta$.

(2a) is trivial. Let us prove (2b). Thus suppose that $I \subseteq \delta, I \in V, p \leq\langle\langle\rangle, \omega\rangle$ and $J \subseteq \omega$ is finite. Let $p=\langle s, A\rangle$.

First we consider the case where $s=\langle\rangle$. Let $a=\emptyset$. We show that $a$ is as required. Thus let $b \subseteq I$ be finite. Let $n \in A$ be such that $n>\max (J)+1$ and $b \subseteq \delta_{n}$. Let $t=s \frown\langle n\rangle$. Note that

$$
\forall \beta_{1} \neq \beta_{2} \in b, \operatorname{range}\left(\underline{\sim}_{1}^{t}\left(\beta_{1}\right)\right) \cap \operatorname{range}\left({\underset{\sim}{p}}_{1}^{t}\left(\beta_{2}\right)\right)=\emptyset .
$$

Let $q=\langle\langle\rangle, B\rangle=\langle\langle\rangle, A \backslash(\max (J)+1)\rangle$. Then $q \leq^{*} p$ and $q$ is compatible with $p_{0}^{t}$. We show that $q$ is as required. We need to show that

(1) $q \|-\forall \beta \in b, \forall k \in J, q_{1}(\beta) \neq k$,

(2) $q \|-\forall \beta_{1} \neq \beta_{2} \in b, \underset{\sim}{q_{1}}\left(\beta_{1}\right) \neq \underset{\sim}{q_{1}}\left(\beta_{2}\right)$.

For (1), if it fails, then we can find $\langle r, D\rangle \leq q, p_{0}^{t}, \beta \in b$ and $k \in J$ such that $\langle r, D\rangle \leq^{*} p_{0}^{r}$ and $\langle r, D\rangle \|-q_{1}(\beta)=k$. But $\langle r, D\rangle \|-q_{1}(\beta)=p_{1}^{r}(\beta)=p_{1}^{t}(\beta)$, and hence $\langle r, D\rangle \|-p_{1}^{t}(\beta)=k$. This is impossible $\operatorname{since} \min (D \widetilde{)} \geq \min \widetilde{(B)}>$ $\max (J)$. For $(2)$, if it fails, then we can find $\langle r, D\rangle \leq q, p_{0}^{t}$ and $\beta_{1} \neq \beta_{2} \in b$ such that $\langle r, D\rangle \leq^{*} p_{0}^{r}$ and $\langle r, D\rangle \|-{\underset{\sim}{q}}_{1}\left(\beta_{1}\right)=\underset{\sim}{q}{ }_{1}\left(\beta_{2}\right)$. As above it follows that 
$\langle r, D\rangle \|-p_{1}^{t}\left(\beta_{1}\right)=p_{1}^{t}\left(\beta_{2}\right)$. This is impossible since for $\beta_{1} \neq \beta_{2} \in b, \operatorname{range}\left(p_{1}^{t}\left(\beta_{1}\right)\right) \cap$ range $\left(p_{1}^{\tau}\left(\beta_{2}\right)\right)=\emptyset$. Hence $q$ is as required and we are done.

Now consider the case $s \neq\langle\rangle$. First we apply (2b) to $t^{s}, I \cap \ln \left(t^{s}\right), p$ and $J$ to find a finite set $a^{\prime} \subseteq \ln \left(t^{s}\right)$ such that

For every finite set $b \subseteq I \cap \operatorname{lh}\left(t^{s}\right) \backslash a^{\prime}$ there is $p^{\prime} \leq^{*} p$ such that $p^{\prime} \|-\left(\forall \beta \in b, \forall k \in J, \underset{\sim}{p_{1}^{s}}(\beta) \neq k\right) \&\left(\forall \beta_{1} \neq\right.$ $\left.\beta_{2} \in b, \underset{\sim}{p}{ }_{1}^{s}\left(\beta_{1}\right) \neq \underset{\sim}{p} s\left(\beta_{2}\right)\right)$.

Let $t^{s}=\left\langle t_{0}, \underset{\sim}{t} 1\right\rangle, \delta_{s(\operatorname{lh}(s)-1)+1} \backslash \delta_{s(\operatorname{lh}(s)-1)}=\left\{\alpha_{J_{1}}, \alpha_{J_{2}}, \ldots\right\}$, where $J_{1}<J_{2}<\ldots$ are in $J_{s(\ln (s)-1)+1}$. Define

$$
a=a^{\prime} \cup\left\{\alpha_{1}, \alpha_{2}, \ldots, \alpha_{J_{\operatorname{lh}(s)+1}}\right\} .
$$

We show that $a$ is as required. First apply (**) to $b \cap \operatorname{lh}\left(t^{s}\right)$ to find $p^{\prime}=\left\langle s, A^{\prime}\right\rangle \leq^{*}$ $p$ such that

$p^{\prime} \|-\left(\forall \beta \in b \cap \operatorname{lh}\left(t^{s}\right), \forall k \in J, \underset{\sim}{t} 1(\beta) \neq k\right) \&\left(\forall \beta_{1} \neq \beta_{2} \in b \cap \operatorname{lh}\left(t^{s}\right), \underset{\sim}{t}{ }_{1}\left(\beta_{1}\right) \neq \underset{\sim}{t}{ }_{1}\left(\beta_{2}\right)\right)$.

Pick $n \in A^{\prime}$ such that $n>\max (J)+1$ and $b \subseteq \delta_{n}$, and let $r=s \frown\langle n\rangle$. Then

$$
\forall \beta_{1} \neq \beta_{2} \in b \backslash \operatorname{lh}\left(t^{s}\right), \operatorname{range}\left({\underset{\sim}{p}}_{1}^{r}\left(\beta_{1}\right)\right) \cap \operatorname{range}\left({\underset{\sim}{p}}_{1}^{r}\left(\beta_{2}\right)\right)=\emptyset .
$$

Pick $k<\omega$ such that $k>n$ and

$$
\forall \beta_{1} \in b \cap \operatorname{lh}\left(t^{s}\right), \forall \beta_{2} \in b \backslash \operatorname{lh}\left(t^{s}\right), \operatorname{range}\left({\underset{\sim}{p}}_{1}^{r}\left(\beta_{1}\right)\right) \cap\left(\operatorname{range}\left({\underset{\sim}{p}}_{1}^{r}\left(\beta_{2}\right)\right) \backslash k\right)=\emptyset .
$$

Let $q=\langle s, B\rangle=\left\langle s, A^{\prime} \backslash(\max (J)+k+1) \cup\{n\}\right\rangle$. Then $q \leq^{*} p^{\prime} \leq^{*} p$ and $q$ is compatible with $p_{0}^{r}$ (since $n \in B$ ). We show that $q$ is as required. We need to prove the following:

(1) $q \|-\forall \beta \in b, \forall k \in J, q_{1}(\beta) \neq k$,

(2) $q \|-\forall \beta_{1} \neq \beta_{2} \in b \backslash \widetilde{\mathrm{h}}\left(t^{s}\right), q_{1}\left(\beta_{1}\right) \neq q_{1}\left(\beta_{2}\right)$,

(3) $q \|-\forall \beta_{1} \in b \cap \operatorname{lh}\left(t^{s}\right), \forall \beta_{2} \in b \backslash \operatorname{lh}\left(t^{s}\right), \underset{\sim}{\sim} \sim_{1}\left(\beta_{1}\right) \neq \underset{\sim}{q} q_{1}\left(\beta_{2}\right)$.

The proofs of (1) and (2) are as in the case $s=\langle\rangle$. Let us prove (3). Suppose that (3) fails. Thus we can find $\langle u, D\rangle \leq q, p_{0}^{r}, \beta_{1} \in b \cap \operatorname{lh}\left(t^{s}\right)$ and $\beta_{2} \in b \backslash \operatorname{lh}\left(t^{s}\right)$ such that $\langle u, D\rangle \leq^{*} p_{0}^{u}$ and $\langle u, D\rangle \|-q_{1}\left(\beta_{1}\right)=q_{1}\left(\beta_{2}\right)$. But for $\beta \in b,\langle u, D\rangle \|-q_{1}(\beta)=$ $p_{1}^{u}(\beta)=p_{1}^{r}(\beta)$, and hence $\langle u, \widetilde{D}\rangle \|-p_{1}^{r}\left(\beta_{1} \widetilde{\tau}=p_{1}^{r}\left(\beta_{2}\right)\right.$. Now note that $\beta_{2} \stackrel{\sim}{=} \alpha_{i}$ for some $i>\widetilde{\mathrm{h}}(s)+1, \min (D) \geq n$ and $\widetilde{\min }(D \backslash\{\widetilde{n}\})>k$. Hence by the construction of $p^{r}$,

$$
\langle u, D\rangle \|-" \underset{\sim}{p}{ }_{1}^{r}\left(\beta_{2}\right) \geq(i-\operatorname{lh}(s)) \text {-th element of } D>k " .
$$

By our choice of $k$, range $\left(\underset{\sim}{p}{ }_{1}^{r}\left(\beta_{1}\right)\right) \cap\left(\operatorname{range}\left({\underset{\sim}{p}}_{1}^{r}\left(\beta_{2}\right)\right) \backslash k\right)=\emptyset$ and we get a contradiction. (3) follows. Thus $\widetilde{q}$ is as required, and the claim follows.

Let

$$
\underset{\sim}{\beta}=\left\{\left\langle p_{0}^{s}, \delta\right\rangle: s \in[\omega]^{<\omega}, \exists \gamma\left(\delta<\gamma, p^{s} \|-\underset{\sim}{\alpha}=\gamma\right)\right\} .
$$

Then $\underset{\sim}{\beta}$ is a $\mathbb{P}_{U}$-name of an ordinal.

Claim 3.12. $\langle\langle\langle\rangle, \omega\rangle, \underset{\sim}{q}\rangle \|-\underset{\sim}{\alpha}=\underset{\sim}{\beta}$.

Proof. Suppose not. There are two cases to be considered. 
Case 1. There are $\left\langle r_{0}, \underset{\sim}{\sim} 1\right\rangle \leq\left\langle\langle\langle\rangle, \omega\rangle, \underset{\sim}{q_{1}}\right\rangle$ and $\delta$ such that $\left\langle r_{0}, \underset{\sim}{\sim}{ }_{1}\right\rangle \|-" \delta \in \underset{\sim}{\alpha} \underset{\sim}{\sim}$ and $\delta \notin \beta$ ". We may suppose that for some ordinal $\alpha,\left\langle r_{0}, \underset{\sim}{\sim}\right\rangle \|-\underset{\sim}{\sim} \stackrel{\sim}{=} \alpha$. Then $\widetilde{\delta}<\alpha$. Let $\widetilde{r}_{0}=\langle s, A\rangle$. Consider $p^{s}=\left\langle p_{0}^{s}, p_{1}^{s}\right\rangle$. Then $p_{0}^{s}$ is compatible with $r_{0}$ and there is a $*$-extension of $p^{s}$ deciding $\underset{\sim}{\alpha}$. Let $t \in N_{s(\ln (s)-1)+1}$ be the $*$-extension of $p^{s}$ deciding $\underset{\sim}{\alpha}$ chosen in the proof of Lemma 3.9. Let $t=\left\langle t_{0}, \underset{\sim}{\sim} 1\right\rangle, t_{0}=\langle s, B\rangle$, and let $\gamma$ be such that $\left\langle t_{0}, \underset{\sim}{\sim} 1\right\rangle \|-\alpha=\gamma$. Let $n \in A \cap B$. Then

- $p_{0}^{s^{\smile}\langle n\rangle}, t_{0}$ and $p_{0}^{s}$ are compatible and $\left\langle s \frown\langle n\rangle, A \cap B \cap A_{s \frown\langle n\rangle}\right\rangle$ extends them, - $p^{s}\langle n\rangle \leq t$.

Thus $p^{s \frown\langle n\rangle} \|-\underset{\sim}{\alpha}=\gamma$. Let $u=\left\langle s \frown\langle n\rangle, A \cap B \cap A_{s \frown\langle n\rangle} \backslash(n+1)\right\rangle$.

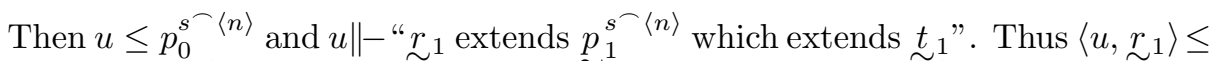
$t,\left\langle r_{0}, \underset{\sim}{r_{1}}\right\rangle, p^{s}\langle n\rangle$. It follows that $\alpha=\gamma$. Now $\delta<\gamma$ and $p^{s-\widetilde{\langle n\rangle}} \|-\underset{\sim}{\alpha}=\gamma$. Hence

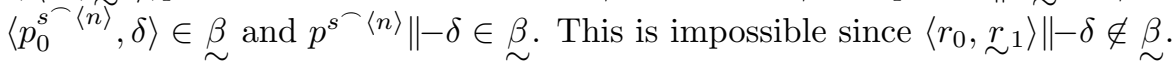

Case 2. There are $\left\langle r_{0},{\underset{\sim}{\sim}}_{1}\right\rangle \leq\left\langle\langle\langle\rangle, \omega\rangle, q_{1}\right\rangle$ and $\delta$ such that $\left\langle r_{0}, \underset{\sim}{\sim}{ }_{1}\right\rangle \|-" \delta \in \beta$ and $\delta \notin \alpha$ ". We may further suppose that for some ordinal $\alpha,\left\langle r_{0}, \underset{\sim}{\sim} \tilde{r}_{1}\right\rangle \|-\alpha=\alpha$. Thus $\delta \geq \sim \alpha$. Let $r=\langle s, A\rangle$. Then as above $p_{0}^{s}$ is compatible with $r$ and there is a *-extension of $p^{s}$ deciding $\underset{\sim}{\alpha}$. Choose $t$ as in Case $1, t=\left\langle t_{0}, \underset{\sim}{\sim} 1\right\rangle, t_{0}=\langle s, B\rangle$ and let $\gamma$ be such that $\left\langle t_{0}, \underset{\sim}{\sim} 1\right\rangle \|-\underset{\sim}{\alpha}=\gamma$. Let $n \in A \cap B$. Then as in Case $1, \alpha=\gamma$ and $p^{s}\langle n\rangle \|-\alpha=\gamma$. On the other hand, since $\left\langle r_{0}, \underset{\sim}{\sim}{ }_{1}\right\rangle \|-\delta \in \underset{\sim}{\beta}$, we can find $\bar{s}$ such that $\bar{s}$ does not contradict $p_{0}^{s^{-}\langle n\rangle},\left\langle p_{0}^{\bar{s}}, p_{1}^{\bar{s}}\right\rangle \|-\underset{\sim}{\alpha}=\bar{\gamma}$, for some $\widetilde{\bar{\gamma}}>\delta$ and $\left\langle p_{0}^{\bar{s}}, \delta\right\rangle \in \underset{\sim}{\sim}$. Now $\bar{\gamma}=\gamma=\alpha>\delta$, which is in contradiction with $\delta \geq \alpha$. The claim follows.

This completes the proof of Lemma 3.9.

Lemma 3.13. Let $\left\langle p_{0},{\underset{\sim}{p}}_{1}\right\rangle \|-f: \omega \longrightarrow O N$. Then there are $\mathbb{P}_{U}$-names $\underset{\sim}{\sim}$ and ${\underset{\sim}{q}}_{1}$ such that $\left\langle p_{0}, \underset{\sim}{q} 1\right\rangle \leq *\left\langle\underset{p_{0}}{\sim} \underset{\sim}{p} 1\right\rangle$ and $\left\langle p_{0}, \underset{\sim}{\sim} 1\right\rangle \|-\underset{\sim}{f}=\underset{\sim}{g}$.

Proof. For simplicity suppose that $\left\langle p_{0}, p_{1}\right\rangle=\langle\langle\langle\rangle, \omega\rangle, \emptyset\rangle$. Let $\theta$ be large enough regular and let $\left\langle N_{n}: n<\omega\right\rangle$ be an increasing sequence of countable elementary submodels of $H_{\theta}$ such that $\mathbb{P}, \underset{\sim}{f} \in N_{0}$ and $N_{n} \in N_{n+1}$ for every $n<\omega$. Let $N=\bigcup_{n<\omega} N_{n}, \delta_{n}=N_{n} \cap \omega_{1}^{\sim}$ for $n<\omega$ and $\delta=\bigcup_{n<\omega} \delta_{n}=N \cap \omega_{1}$. Let $\left\langle J_{n}: n<\omega\right\rangle \in N_{0}$ and $\left\langle\alpha_{i}: 0<i<\omega\right\rangle$ be as in Lemma 3.9.

We define by induction a sequence $\left\langle p^{s}: s \in[\omega]^{<\omega}\right\rangle$ of conditions and a sequence $\left\langle\sim_{s}^{\beta}: s \in[\omega]^{<\omega}\right\rangle$ of $\mathbb{P}_{U}$-names for ordinals such that

- $p^{s}=\left\langle p_{0}^{s}, \underset{\sim}{p}{ }_{1}^{s}\right\rangle=\left\langle\langle s, \omega \backslash(\max (s)+1)\rangle,{\underset{\sim}{p}}_{1}^{s}\right\rangle$,

- $p^{s} \in N_{s(\ln (s)-1)+1}$,

- $\operatorname{lh}\left(p^{s}\right) \geq \delta_{s(\operatorname{lh}(s)-1)}$,

- $p^{s} \|-" f(\operatorname{lh}(s)-1)=\beta_{s}$ ",

- if $t$ end extends $s$, then $p^{t} \leq p^{s}$.

For $s=\langle\rangle$, let $p^{\langle\rangle}=\langle\langle\langle\rangle, \omega\rangle, \emptyset\rangle$. Now suppose that $s \neq\langle\rangle$ and $p^{s \mid \ln (s)-1}$ is defined. We define $p^{s}$. Let $C_{s \uparrow \ln (s)-1}$ be an infinite subset of $\omega$ almost disjoint to $\left\langle\operatorname{range}\left(p_{1}^{s\lceil\operatorname{lh}(s)-1}(\beta)\right): \beta<\operatorname{lh}\left(p^{s\lceil\operatorname{lh}(s)-1}\right)\right\rangle$. Split $C_{s \uparrow \operatorname{lh}(s)-1}$ into $\omega$ infinite disjoint sets $\left\langle C_{s \uparrow \mathrm{hh}(s)-1, t}: t \in[\omega]^{<\omega}\right.$ and the $t$ end extends $\left.s \uparrow \operatorname{lh}(s)-1\right\rangle$. Again split $C_{s \uparrow \operatorname{lh}(s)-1, s}$ into $\omega$ infinite disjoint sets $\left\langle C_{i}: i<\omega\right\rangle$. Let $\left\langle c_{i j}: j<\omega\right\rangle$ be an increasing enumeration of $C_{i}, i<\omega$. We may suppose that all of these are done in $N_{s(\operatorname{lh}(s)-1)+1}$. Let $q^{s}=\left\langle q_{0}^{s}, \underset{\sim}{q}{ }_{1}^{s}\right\rangle$, where

- $q_{0}^{s}=\langle s, \omega \backslash(\max (s)+1)\rangle$,

- for $\beta<\operatorname{lh}\left(p^{s\lceil\operatorname{lh}(s)-1}\right), \underset{\sim}{q}{ }_{1}^{s}(\beta)={\underset{\sim}{p}}_{1}^{s \uparrow \operatorname{lh}(s)-1}(\beta)$, 
- for $i \in J_{s(\operatorname{lh}(s)-1)}$ such that $\alpha_{i} \in \delta_{s(\operatorname{lh}(s)-1)} \backslash \operatorname{lh}\left(p^{s\lceil\operatorname{lh}(s)-1}\right)$,

$$
\underset{1}{q}\left(\alpha_{i}\right)=\left\{\left\langle\left\langle s \frown\left\langle r_{1}, \ldots, r_{i}\right\rangle, \omega \backslash\left(r_{i}+1\right)\right\rangle, c_{i r_{i}}\right\rangle: r_{1}>\max (s),\left\langle r_{1}, \ldots, r_{i}\right\rangle \in\right.
$$

Then $q^{s} \in N_{s(\operatorname{lh}(s)-1)+1}$ and as in the proof of claim 3.10, $q^{s} \in \mathbb{P}$. By Lemma 3.9, applied inside $N_{s(\operatorname{lh}(s)-1)+1}$, we can find $\mathbb{P}_{U}$-names $\beta_{s}$ and ${\underset{\sim}{s}}_{1}^{s}$ such that $\left\langle q_{0}^{s},{\underset{\sim}{1}}_{1}^{s}\right\rangle \leq$ $\left\langle q_{0}^{s}, q_{1}^{s}\right\rangle$ and $\left\langle q_{0}^{s}, \underset{\sim}{p} p_{1}^{s}\right\rangle \|-\underset{\sim}{f}(\operatorname{lh}(s)-1)=\beta_{s}$. Let $p^{\widetilde{s}}=\left\langle p_{0}^{s}, \underset{\sim}{\sim} \sim_{1}^{s}\right\rangle=\left\langle q_{0}^{s}, \underset{\sim}{p_{1}^{s}}\right\rangle$. Then $p^{s} \leq p^{s \uparrow \ln (s)-1}$ and $p^{s} \|-\widetilde{f}\left\lceil\ln (s)=\left\{\left\langle i, \widetilde{\beta}_{s \uparrow i+1}\right\rangle: i<\operatorname{lh}(s)\right\}\right.$.

This completes our definition of the sequences $\left\langle p^{s}: s \in[\omega]<\omega\right\rangle$ and $\left\langle\beta_{s}: s \in\right.$ $\left.[\omega]^{<\omega}\right\rangle$. Let

$$
\begin{aligned}
& \underline{\sim}_{1}=\left\{\left\langle p_{0}^{s},\left\langle\beta, \underset{\sim}{p_{1}^{s}}(\beta)\right\rangle\right\rangle: s \in[\omega]^{<\omega}, \beta<\operatorname{lh}\left(p^{s}\right)\right\}, \\
& \underset{\sim}{g}=\left\{\left\langle p_{0}^{s},\left\langle i,{\underset{\sim}{\beta}}_{s \mid i+1}\right\rangle\right\rangle: s \in[\omega]^{<\omega}, i<\operatorname{lh}(s)\right\} .
\end{aligned}
$$

Then $\underset{\sim}{\sim}{ }_{1}$ and $\underset{\sim}{g}$ are $\mathbb{P}_{U}$-names.

Claim 3.14. $\left\langle\langle\langle\rangle, \omega\rangle, \underset{\sim}{q_{1}}\right\rangle \in \mathbb{P}$.

Proof. We check conditions in Definition 3.4.

(1) i.e. $\langle\langle\rangle, \omega\rangle \in \mathbb{P}_{U}$ is trivial.

(2) It is clear by our construction that

$$
\langle\langle\rangle, \omega\rangle \|-"{\underset{\sim}{q}}_{1} \text { is a well-defined function" }
$$

and as in the proof of Claim 3.11, we can show that $\operatorname{lh}\left(q_{1}\right)=\delta$. (2a) is trivial. Let us prove (2b). Thus suppose that $I \subseteq \delta, I \in V, p \leq\langle\langle\rangle, \omega)$ and $J \subseteq \omega$ is finite. Let $p=\langle s, A\rangle$. If $s=\langle\rangle$, then as in the proof of Claim 3.11, we can show that $a=\emptyset$ is a required. Thus suppose that $s \neq\langle\rangle$. First we apply (2b) to $p^{s}, I \cap \operatorname{lh}\left(p^{s}\right), p$ and $J$ to find $a^{\prime} \subseteq \operatorname{lh}\left(p^{s}\right)$ such that

For every finite $b \subseteq I \cap \ln \left(p^{s}\right) \backslash a^{\prime}$ there is $p^{\prime} \leq^{*} p$ such that $p^{\prime} \|-\left(\forall \beta \in b, \forall k \in J,{\underset{\sim}{\sim}}_{1}^{s}(\beta) \neq k\right) \&\left(\forall \beta_{1} \neq \beta_{2} \in\right.$ $\left.b, \underset{\sim}{p}{ }_{1}^{s}\left(\beta_{1}\right) \neq \underset{\sim}{p} p_{1}^{s}\left(\beta_{2}\right)\right)$.

Let $\delta_{s(\operatorname{lh}(s)-1)+1} \backslash \delta_{s(\operatorname{lh}(s)-1)}=\left\{\alpha_{J_{1}}, \ldots, \alpha_{J_{i}}, \ldots\right\}$ where $J_{1}<J_{2}<\ldots$ are in $J_{s(\ln (s)-1)+1}$. Let

$$
a=a^{\prime} \cup\left\{\alpha_{1}, \alpha_{2}, \ldots, \alpha_{J_{\mathrm{lh}(s)}}\right\}
$$

We show that $a$ is as required. Let $b \subseteq I \backslash a$ be finite. First we apply $(*)$ to $b \cap \operatorname{lh}\left(p^{s}\right)$ to find $p^{\prime}=\left\langle s, A^{\prime}\right\rangle \leq^{*} p$ such that

$p^{\prime} \|-\left(\forall \beta \in b \cap \operatorname{lh}\left(p^{s}\right), \forall k \in J,{\underset{\sim}{p}}_{1}^{s}(\beta) \neq k\right) \&\left(\forall \beta_{1} \neq \beta_{2} \in b \cap \operatorname{lh}\left(p^{s}\right),{\underset{\sim}{p}}_{1}^{s}\left(\beta_{1}\right) \neq{\underset{\sim}{p}}_{1}^{s}\left(\beta_{2}\right)\right)$.

Also note that for $\beta \in b \cap \operatorname{lh}\left(p^{s}\right), p^{\prime} \|-q_{1}(\beta)=p_{1}^{s}(\beta)$. Pick $m$ such that $\max (s)+$ $\max (J)+1<m<\omega$, and if $t$ end extends $s$ and $m<\max (t)$, then $C_{s, t}$ is disjoint to $J$ and to range $\left(p_{1}^{s}(\beta)\right)$ for $\beta \in b \cap \operatorname{lh}\left(p^{s}\right)$. Then pick $n>m, n \in A^{\prime}$ such that $b \subseteq \delta_{n}$, and let $t=\widetilde{s} \frown\langle n\rangle$. Then

- $\forall \beta_{1} \neq \beta_{2} \in b \backslash \operatorname{lh}\left(p^{s}\right)$, range $\left(p_{1}^{t}\left(\beta_{1}\right)\right) \cap \operatorname{range}\left(p_{1}^{t}\left(\beta_{2}\right)\right)=\emptyset$,

- $\forall \beta_{1} \in b \cap \operatorname{lh}\left(p^{s}\right), \forall \beta_{2} \in b \backslash \operatorname{lh}\left(\widetilde{p}^{s}\right)$, range $\left({\underset{\sim}{p}}_{1}^{t}\left(\beta_{1}\right)\right) \cap \operatorname{range}\left({\underset{\sim}{p}}_{1}^{t}\left(\beta_{2}\right)\right)=\emptyset$,

- $\forall \beta \in b \backslash \operatorname{lh}\left(p^{s}\right)$, range $(\underset{\sim}{p} t(\beta)) \cap J=\emptyset$. 
Let $q=\langle s, B\rangle=\left\langle s, A^{\prime} \backslash(n+1)\right\rangle$. Then $q \leq^{*} p^{\prime} \leq^{*} p$ and using the above facts we can show that

$$
\begin{aligned}
& q \|-\left(\forall \beta \in b, \forall k \in J, q_{\sim}^{q}(\beta)={\underset{\sim}{p}}_{1}^{t}(\beta) \neq k\right) \&\left(\forall \beta_{1} \neq \beta_{2} \in b, \underset{\sim}{q_{1}}\left(\beta_{1}\right)={\underset{\sim}{p}}_{1}^{t}\left(\beta_{1}\right)\right. \\
& \left.\neq \underset{\sim}{p_{1}^{t}}\left(\beta_{2}\right)={\underset{\sim}{q}}_{1}\left(\beta_{2}\right)\right) \text {. }
\end{aligned}
$$

Thus $q$ is as required and the claim follows.

Claim 3.15. $\left\langle\langle\langle\rangle, \omega\rangle, \underset{\sim}{\sim} q_{1}\right\rangle \|-\underset{\sim}{f}=\underset{\sim}{\sim}$.

Proof. Suppose not. Then we can find $\left\langle r_{0},{\underset{\sim}{\sim}}_{1}\right\rangle \leq\left\langle\langle\langle\rangle, \omega\rangle, q_{1}\right\rangle$ and $i<\omega$ such that $\left\langle r_{0},{\underset{\sim}{\sim}}_{1}\right\rangle \|-f(i) \neq g(i)$. Let $r_{0}=\langle s, A\rangle$. Then $r_{0}$ is compationle with $p_{0}^{s}$ and $r_{0} \|-{ }^{*} r_{1}$

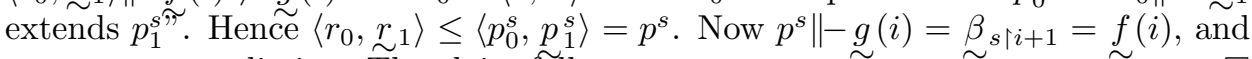
we get a contradiction. The claim follows.

This completes the proof of Lemma 3.13.

The following is now immediate.

Lemma 3.16. The forcing $(\mathbb{P}, \leq)$ preserves cofinalities.

Proof. By Lemma 3.13, $\mathbb{P}$ preserves cofinalities $\leq \omega_{1}$. On the other hand, by a $\Delta$ system argument, $\mathbb{P}$ satisfies the $\omega_{2}$-c.c. and hence it preserves cofinalities $\geq \omega_{2}$.

Lemma 3.17. Let $G$ be $(\mathbb{P}, \leq)$-generic over $V$. Then $V[G] \models G C H$.

Proof. By Lemma 3.13, $V[G] \models C H$. Now let $\kappa \geq \omega_{1}$. Then

$$
\left(2^{\kappa}\right)^{V[G]} \leq\left(\left(|\mathbb{P}|^{\omega_{1}}\right)^{\kappa}\right)^{V} \leq\left(2^{\kappa}\right)^{V}=\kappa^{+}
$$

The result follows.

Now we return to the proof of Theorem 3.1. Suppose that $G$ is $(\mathbb{P}, \leq)$-generic over $V$, and let $V_{1}=V[G]$. Then $V_{1}$ is a cofinality and $G C H$ preserving generic extension of $V$. We show that adding a Cohen real over $V_{1}$ produces $\aleph_{1}$-many Cohen reals over $V$. Thus force to add a Cohen real over $V_{1}$. Split it into $\omega$ Cohen reals over $V_{1}$. Denote them by $\left\langle r_{n, m}: n, m<\omega\right\rangle$. Also let $\left\langle f_{i}: i<\omega_{1}\right\rangle \in V$ be a sequence of almost disjoint functions from $\omega$ into $\omega$. First we define a sequence $\left\langle s_{n, i}: i<\omega_{1}\right\rangle$ of reals by

$$
\forall k<\omega, s_{n, i}(k)=r_{n, f_{i}(k)}(0) .
$$

Let $\left\langle I_{n}: n<\omega\right\rangle$ be the partition of $\omega_{1}$ produced by $G$. For $\alpha<\omega_{1}$ let

- $n(\alpha)=$ that $n<\omega$ such that $\alpha \in I_{n}$,

- $i(\alpha)=$ that $i<\omega_{1}$ such that $\alpha$ is the $i$-th element of $I_{n(\alpha)}$.

We define a sequence $\left\langle t_{\alpha}: \alpha<\omega_{1}\right\rangle$ of reals by $t_{\alpha}=s_{n(\alpha), i(\alpha)}$. The following lemma completes the proof of Theorem 3.1.

Lemma 3.18. $\left\langle t_{\alpha}: \alpha<\omega_{1}\right\rangle$ is a sequence of $\aleph_{1}$-many Cohen reals over $V$.

Proof. First note that $\left\langle r_{n, m}: n, m<\omega\right\rangle$ is $\mathbb{C}(\omega \times \omega)$-generic over $V_{1}$. By the c.c.c. of $\mathbb{C}\left(\omega_{1}\right)$ it suffices to show that for every countable $I \subseteq \omega_{1}, I \in V,\left\langle t_{\alpha}: \alpha \in I\right\rangle$ is $\mathbb{C}(I)$-generic over $V$. Thus it suffices to prove the following:

For every $\left\langle\left\langle p_{0}, p_{1}\right\rangle, q\right\rangle \in \mathbb{P} * \mathbb{C}(\omega \times \omega)$ and every open dense subset $D^{\sim} \in V$ of $\mathbb{C}(I)$, there is $\left\langle\left\langle q_{0}, q_{1}\right\rangle, r\right\rangle \leq$

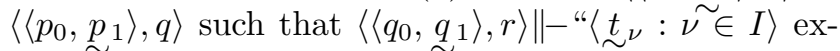
tends some element of $D$ ". 
Let $\left\langle\left\langle p_{0}, p_{1}\right\rangle, q\right\rangle$ and $D$ be as above. Let $\alpha=\sup (I)$. We may suppose that $\operatorname{lh}\left(\underline{\sim}_{1}\right) \geq \alpha$. Let $J=\{n: \exists m, k,\langle n, m, k\rangle \in \operatorname{dom}(q)\}$. We apply (2b) to $\left\langle p_{0}, \underline{\sim}_{1}\right\rangle$, $I, \widetilde{p_{0}}$ and $J$ to find a finite set $a \subseteq I$ such that:

For every finite $b \subseteq I \backslash a$ there is $p_{0}^{\prime} \leq^{*} p_{0}$ such

(**) $\quad$ that $p_{0}^{\prime} \|-\left(\forall \beta \in b, \forall k \in J, \underset{\sim}{p_{1}}(\beta) \neq k\right) \&\left(\forall \beta_{1} \neq \beta_{2} \in\right.$ $\left.b, \underset{\sim}{p}{ }_{1}\left(\beta_{1}\right) \neq \underset{\sim}{p} p_{1}\left(\beta_{2}\right)\right)$.

Let

$$
S=\left\{\langle\nu, k, j\rangle: \nu \in a, k<\omega, j<2,\left\langle n(\nu), f_{i(\nu)}(k), 0, j\right\rangle \in q\right\} .
$$

Then $S \in \mathbb{C}\left(\omega_{1}\right)$. Pick $k_{0}<\omega$ such that for all $\nu_{1} \neq \nu_{2} \in a$, and $k \geq k_{0}$, $f_{i\left(\nu_{1}\right)}(k) \neq f_{i\left(\nu_{2}\right)}(k)$. Let

$$
S^{*}=S \cup\left\{\langle\nu, k, 0\rangle: \nu \in a, k<\kappa_{0},\langle\nu, k, 1\rangle \notin S\right\} .
$$

The reason for defining $S^{*}$ is to avoid possible collisions. Then $S^{*} \in \mathbb{C}\left(\omega_{1}\right)$. Pick $S^{* *} \in D$ such that $S^{* *} \leq S^{*}$. Let $b=\left\{\nu: \exists k, j,\langle\nu, k, j\rangle \in S^{* *}\right\} \backslash q$. By $(* *)$ there is $p_{0}^{\prime} \leq^{*} p_{0}$ such that

$$
p_{0}^{\prime} \|-\left(\forall \nu \in b, \forall k \in J, \underset{\sim}{p_{1}}(\nu) \neq k\right) \&\left(\forall \nu_{1} \neq \nu_{2} \in b, \underset{\sim}{p_{1}}\left(\nu_{1}\right) \neq{\underset{\sim}{p}}_{1}\left(\nu_{2}\right)\right) .
$$

Let $p_{0}^{\prime \prime} \leq p_{0}^{\prime}$ be such that $\left\langle p_{0}^{\prime \prime}, \underset{\sim}{p_{1}}\right\rangle$ decides all the colors of elements of $a \cup b$. Let

$$
q^{*}=q \cup\left\{\left\langle n(\nu), f_{i(\nu)}(k), 0, S^{* *}(\nu, k)\right\rangle:\langle\nu, k\rangle \in \operatorname{dom}\left(S^{* *}\right)\right\} .
$$

Then $q^{*}$ is well defined and $q^{*} \in \mathbb{C}(\omega \times \omega)$. Now $q^{*} \leq q,\left\langle\left\langle p_{0}^{\prime \prime}, \underset{\sim}{p_{1}}\right\rangle, q^{*}\right\rangle \leq\left\langle\left\langle p_{0}, \underset{\sim}{p} p_{1}\right\rangle, q\right\rangle$, and for $\langle\nu, k\rangle \in \operatorname{dom}\left(S^{* *}\right)$,

$$
\left\langle\left\langle p_{0}^{\prime \prime}, \underset{\sim}{p_{1}}\right\rangle, q^{*}\right\rangle \|-S^{* *}(\nu, k)=q^{*}\left(n(\nu), f_{i(\nu)}(k), 0\right)=\underset{\sim}{\sim} n(\nu), f_{i(\nu)}(k)(0)=\underset{\sim}{t} \nu(k) .
$$

It follows that

$$
\left\langle\left\langle p_{0}^{\prime \prime}, \underset{\sim}{p_{1}}\right\rangle, q^{*}\right\rangle \|-“\langle\underset{\sim}{\sim} \nu: \nu \in I\rangle \text { extends } S^{* *} "
$$

(*) and hence Lemma 3.18 follow.

\section{ACKNOWLEDGEMENT}

The authors would like to thank the anonymous referee for carefully reading the paper and offering extensive improvement to its exposition.

\section{REFERENCES}

[Cu] James Cummings, A model in which GCH holds at successors but fails at limits, Trans. Amer. Math. Soc. 329 (1992), no. 1, 1-39, DOI 10.2307/2154075. MR1041044 (92h:03076)

[D-J1] A. J. Dodd and R. B. Jensen, The covering lemma for $L[U]$, Ann. Math. Logic 22 (1982), no. 2, 127-135, DOI 10.1016/0003-4843(82)90018-3. MR667224(83i:03082b)

[D-J2] Tony Dodd and Ronald Jensen, The covering lemma for $K$, Ann. Math. Logic 22 (1982), no. 1, 1-30, DOI 10.1016/0003-4843(82)90013-4. MR661475 (83i:03082a)

[Git1] MM. Gitik, Adding a lot of Cohen reals by adding a few, Unpublished paper.

[Git2] Moti Gitik, Prikry-type forcings, Handbook of set theory. Vols. 1, 2, 3, Springer, Dordrecht, 2010, pp. 1351-1447, DOI 10.1007/978-1-4020-5764-9_17. MR2768695

[Git-Mag1] Moti Gitik and Menachem Magidor, The singular cardinal hypothesis revisited, Set theory of the continuum (Berkeley, CA, 1989), Math. Sci. Res. Inst. Publ., vol. 26, Springer, New York, 1992, pp. 243-279, DOI 10.1007/978-1-4613-9754-0_16. MR $1233822(95 \mathrm{c}: 03131)$ 
[Git-Mag2] Moti Gitik and Menachem Magidor, Extender based forcings, J. Symbolic Logic 59 (1994), no. 2, 445-460, DOI 10.2307/2275399. MR.1276624(95k:03079)

[G-N-S] Moti Gitik, Itay Neeman, and Dima Sinapova, A cardinal preserving extension making the set of points of countable $V$ cofinality nonstationary, Arch. Math. Logic 46 (2007), no. 5-6, 451-456, DOI 10.1007/s00153-007-0048-0. MR2321586 (2008e:03085)

[Mag1] Menachem Magidor, Changing cofinality of cardinals, Fund. Math. 99 (1978), no. 1, 61-71. MR0465868 (57 \#5754)

[Mag2] Menachem Magidor, How large is the first strongly compact cardinal? or A study on identity crises, Ann. Math. Logic 10 (1976), no. 1, 33-57. MR0429566 (55 \#2578)

[Mer] Carmi Merimovich, Prikry on extenders, revisited, Israel J. Math. 160 (2007), 253280, DOI 10.1007/s11856-007-0063-1. MR 2342498(2008j:03075)

[Sh1] Saharon Shelah, Proper forcing, Lecture Notes in Mathematics, vol. 940, SpringerVerlag, Berlin, 1982. MR675955 (84h:03002)

[Sh2] Saharon Shelah, Cardinal arithmetic, Oxford Logic Guides, vol. 29, The Clarendon Press Oxford University Press, New York, 1994. Oxford Science Publications. MR 1318912 (96e:03001)

[Ve-W] Boban Velickovic and W. Hugh Woodin, Complexity of reals in inner models of set theory, Ann. Pure Appl. Logic 92 (1998), no. 3, 283-295, DOI 10.1016/S01680072(98)00010-4. MR 1640916 (99f:03067)

School of Mathematical Sciences, Tel Aviv University, Tel Aviv, Israel

E-mail address: gitik@post.tau.ac.il

Kurt Gödel Research Center for Mathematical Logic (KGRC), Vienna, Austria

E-mail address: golshani.m@gmail.com 